\title{
Learning Probabilistic Read-once Formulas on Product Distributions
}

ROBERT E. SCHAPIRE

SCHAPIRE@RESEARCH.ATT.COM

ATET Bell Laboratories, 600 Mountain Avenue, Room 2A-424, Murray Hill, NJ 07974

Editors: Ming Li and Leslie Valiant

\begin{abstract}
This paper presents a polynomial-time algorithm for inferring a probabilistic generalization of the class of read-once Boolean formulas over the usual basis $\{$ AND, OR, NOT\}. The algorithm effectively infers a good approximation of the target formula when provided with random examples which are chosen according to any product distribution, i.e., any distribution in which the setting of each input bit is chosen independently of the settings of the other bits. Since the class of formulas considered includes ordinary read-once Boolean formulas, our result shows that such formulas are PAC learnable (in the sense of Valiant) against any product distribution (for instance, against the uniform distribution). Further, this class of probabilistic formulas includes read-once formulas whose behavior has been corrupted by large amounts of random noise. Such noise may affect the formula's output ("misclassification noise"), the input bits ("attribute noise"), or it may affect the behavior of individual gates of the formula. Thus, in this setting, we show that read-once formula's can be inferred (approximately), despite large amounts of noise affecting the formula's behavior.
\end{abstract}

Keywords: computational learning theory, PAC-learning, learning with noise, read-once formulas, product distributions

\section{Introduction}

This paper describes an algorithm for learning a probabilistic generalization of the class of read-once formulas over the usual basis $\{$ AND, OR, NOT\}. (A formula is read-once if each variable appears at most once in the formula.) This algorithm is based on a statistical technique for discovering the structure of a read-once formula. An especially nice feature of this technique is its powerful resistance to noise or randomness.

Similar to the Valiant (1984) model, we consider the problem of learning from randomly chosen examples. We apply our method to a probabilistic generalization of the class of all read-once Boolean formulas constructed from the usual basis \{AND, OR, NOT\}. We show that an arbitrarily good approximation of such formulas can be inferred in polynomial time against any product distribution (i.e., any distribution in which the setting of each variable is chosen independently of the settings of the other variables). For example, this shows that the class of read-once Boolean formulas over the usual basis can be learned in polynomial time against the uniform distribution in the sense of Valiant. This contrasts sharply with the 
results of Kearns and Valiant (1989) which show that Boolean formulas, even if read-once, cannot be learned against arbitrary distributions.

The problem of learning Boolean formulas against special distributions has been considered by a number of other authors. In particular, our technique closely resembles that used by Goldman, Kearns and Schapire (1990) for exactly identifying certain classes of read-once formulas when the observed examples are chosen randomly according to specific, fixed and simple distributions. This technique also resembles that of Kearns et al. (1987) for learning the class of read-once formulas in disjunctive normal form (DNF) against the uniform distribution. A similar result, though based on a different method, was obtained by Pagallo and Haussler (1989). Our results extend theirs to a much broader class of read-once formulas. Their results were also extended in a different direction by Hancock and Mansour (1991).

Linial, Mansour and Nisan (1989) used a technique based on Fourier spectra to learn the class of constant-depth formulas (constructed from gates of unbounded fan-in) against the uniform distribution. Furst, Jackson and Smith (1991) generalized this result to learn this same class against any product distribution. Verbeurgt (1990) gives a different algorithm for learning DNF-formulas against the uniform distribution. However, all three of these algorithms require quasi-polynomial $\left(n^{\text {polylog(n)}}\right)$ time, though Verbeurgt's procedure only requires a polynomial-size sample.

The class of read-once Boolean formulas has been considered previously by a number of authors. Angluin, Hellerstein and Karpinski (1993) proved that this class of formulas can be exactly identified using membership and equivalence queries. This result has since been extended to much broader bases by Hancock (1990), Hellerstein and Karpinski (1990), and Hancock and Hellerstein (1991). Particularly relevant is the algorithm given by these latter authors for learning read-once formulas over fields in which the gate operations are addition and multiplication; their algorithm bears very strong resemblance to the one presented in the current paper. Their work was recently extended by Bshouty, Hancock and Hellerstein (1992) to also handle division gates.

We adopt from Kearns and Schapire (1990) the notion of a probabilistic concept ( $p$-concept). (This learning model is essentially equivalent to Yamanishi's (1992) model for learning "stochastic rules.") A p-concept $c$ is a function that maps each input-variable assignment $x$ to a real number $c(x)$ between 0 and 1 . We interpret $c(x)$ as the probability that instance $x$ will be positively classified. Thus, in the pconcept model, a randomly labeled example is chosen as follows: first, an instance $x$ is chosen at random according to the target distribution on the instance space; then, with probability $c(x)$, the labeled example $(x, 1)$ is observed, and with probability $1-c(x)$, the labeled example $(x, 0)$ is observed. Thus, in general, the learner has no direct access to the function $c$, even on individual points.

We view the learning problem as that of inferring from such randomly chosen examples a good approximation of the function $c$ itself. Thus, we ask that the learner infer a real-valued hypothesis $h$ for which $|h(x)-c(x)|$ is small for most instances $x$. This is called learning with a model of probability. 
Specifically, we consider the problem of learning a class of real-valued read-once formulas, called read-once real formulas. Formulas in this class are constructed using two kinds of gates, or operators: The first gate, denoted MUL, simply computes the product of its real-valued inputs. The second gate, $\operatorname{LIN}_{z ; w_{1}, \ldots, w_{k}}$, computes the weighted sum

$$
\operatorname{LIN}_{z ; w_{1}, \ldots, w_{k}}\left(y_{1}, \ldots, y_{k}\right)=z+w_{1} y_{1}+\cdots+w_{k} y_{k} .
$$

Here, $k$ may be any positive integer, and $z$ and the $w_{i}$ 's may be any real numbers which yield a function $\operatorname{LIN}_{z ; w_{1}, \ldots, w_{k}}$ whose range is $[0,1]$ on the domain $[0,1]^{k}$.

Clearly, for a Boolean assignment to the input variables, a formula constructed from such gates outputs a real number between 0 and 1 , and so these are indeed p-concepts. We show that this class can be learned with a model of probability against any product distribution (such as the uniform distribution).

Note that, for Boolean-valued inputs, the function MUL simply computes the logical AND of its inputs, and $\operatorname{LiN}_{1 ;-1}$ computes the logical negation of its single input. Thus, the class of read-once real formulas includes the class of read-once Boolean formulas with basis \{AND, OR, NOT\}. Therefore, our result demonstrates for the first time the existence of a polynomial-time algorithm for inferring a good approximation of any such Boolean formula (against a product distribution).

Also, a unary gate $\operatorname{LIN}_{z ; w}$ can alternatively be viewed as describing the behavior of a noisy or random Boolean gate which, on input 0 randomly outputs 1 with probability $z$, and on input 1 outputs 1 with probability $z+w$. (If the input to such a randomized gate is 1 with probability $p$, then the output is easily computed to be 1 with probability $\operatorname{LIN}_{z ; w}(p)=z+w p$.) Thus, for the distributions considered, our result can be regarded as a demonstration of the learnability of read-once Boolean formulas, even when every gate and every wire of the formula is corrupted by significant amounts of randomness.

A similar probabilistic interpretation can be given to a non-unary gate $\operatorname{LIN}_{z ; w_{1}, \ldots, w_{k}}$. Specifically, we can view such a gate as describing the probabilistic behavior of a kind of random "multiplexor" gate that, on inputs $y_{1}, \ldots, y_{k}$, does the following:

- with probability $w_{i}$, the gate outputs $y_{i}$;

- with probability $z$, the gate ignores its inputs and simply outputs 1 ;

- and with probability $1-z-\sum w_{i}$, the gate ignores its inputs and outputs 0 .

Thus, if input $y_{i}$ is 1 with probability $p_{i}$, then the probability that such a randomized gate outputs 1 is $z+\sum w_{i} p_{i}$.

For instance, the binary gate $\operatorname{LIN}_{0 ; .5, .5}$ describes the behavior of a randomized gate that flips a fair coin and, if "heads" evaluates (and outputs) the left input, and if "tails" evaluates the right input.

Our result can therefore be viewed as a proof of the learnability of Boolean formulas constructed from the usual Boolean gates, in addition to the stochastic gates just described. 


\section{Preliminaries}

In this paper, we will be exclusively interested in the learning of read-once formulas $f$ constructed from gates (chosen from some basis), variables (denoted $x_{1}, \ldots, x_{n}$, and assumed in this paper to be Boolean) and, possibly, constants. We use the term node to refer to either a gate or a variable of some formula $f$. Note that because we consider only read-once formulas, there is a unique path from any node of $f$ to the output. We define the level or depth of a node $\lambda$ to be the number of gates (not including $\lambda$ itself) on the path from $\lambda$ to the output. Thus, the output node is at level 0 . The depth of the entire formula is the maximum level of any node.

We say that a node $\lambda$ feeds a gate $\lambda^{\prime}$ if the path from $\lambda$ to the output goes through $\lambda^{\prime}$. If $\lambda$ is an input to $\lambda^{\prime}$, then we say that $\lambda$ immediately feeds $\lambda^{\prime}$. For any two variables $x_{i}$ and $x_{j}$ we define $\Gamma_{i j}$ to be the deepest gate $\lambda$ fed by both $x_{i}$ and $x_{j}$. We say that a pair of variables $x_{i}$ and $x_{j}$ meet at the gate $\Gamma_{i j}$. We also write $g_{i j}$ to denote the subformula subsumed by gate $\Gamma_{i j}$. (A subformula $g$ is subsumed by node $\lambda$ if $\lambda$ is the root or output node of $g$.)

For $q_{j} \in\{0,1\}$ and $i_{j} \in\{1, \ldots, n\}, 1 \leq j \leq r$, we write $f \mid x_{i_{1}} \leftarrow q_{1}, \ldots, x_{i_{r}} \leftarrow q_{r}$ to denote the function obtained from $f$ by fixing or hard-wiring each variable $x_{i_{j}}$ to the value $q_{j}$.

In our framework, the learner is attempting to infer an unknown target concept $c$ chosen from some known concept class $\mathcal{C}$. In this paper, $\mathcal{C}=\bigcup_{n \geq 1} \mathcal{C}_{n}$ is parameterized by the number of variables $n$, and each $c \in \mathcal{C}_{n}$ represents a Boolean function on the domain $\{0,1\}^{n}$.

In the distribution-free or probably approximately correct (PAC) learning model, introduced by Valiant (1984), the learner is given access to labeled (positive and negative) examples of the target concept, drawn randomly and independently according to some unknown target distribution $D$. The learner is also given as input positive real numbers $\epsilon$ and $\delta$. The learner's goal is to output with probability at least $1-\delta$ a hypothesis $h$ that has probability at most $\epsilon$ of disagreeing with $c$ on a randomly drawn example from $D$ (thus, the hypothesis has accuracy at least $1-\epsilon$, and is said to be $\epsilon$-good). If such a learning algorithm exists (that is, a polynomialtime algorithm meeting the goal for any $n \geq 1$, any $c \in \mathcal{C}_{n}$, any distribution $D$, and any positive values of $\epsilon$ and $\delta$ ), we say that $\mathcal{C}$ is $P A C$-learnable. In this setting, polynomial time means polynomial in $n, 1 / \epsilon$ and $1 / \delta$. In this paper, we will be primarily interested in a variant of Valiant's model in which the target distribution is known a priori to belong to a specific restricted class of distributions.

As described in detail by Kearns and Schapire (1990), a probabilistic concept ( $p$ concept) is a function $c:\{0,1\}^{n} \rightarrow[0,1]$. The interpretation here is that $c(x)$ is the probability that an instance $x \in\{0,1\}^{n}$ is labeled 1 , and $1-c(x)$ is the probability it is labeled 0 . Thus, we assume an oracle $E X$ that first chooses $x$ randomly according to some target distribution $D$, and then randomly labels $x$ according to $c$ as just described.

In this paper, we will be interested in the problem of learning with a model of probability. Here, the goal is to infer a good approximation of the function $c$ itself. 
Specifically, given positive $\epsilon$ and $\delta$, we ask that, with probability at least $1-\delta$, the learning algorithm find an $\epsilon$-good model of probability for $f$, i.e., a real-valued hypothesis $h$ such that

$$
\mathbf{E}_{x \in D}[\mid h(x)-c(x) \|] \leq \epsilon .
$$

Furthermore, the learning algorithm's running time must be polynomial in $1 / \epsilon, 1 / \delta$ and $n$. (This definition differs slightly, but is equivalent to, the definition of learning with a model of probability given by Kearns and Schapire (1990).)

We describe an algorithm for learning with a model of probability any p-concept in a particular class of p-concepts against any product distribution on the domain $\{0,1\}^{n}$, i.e., any distribution in which the setting of each bit $x_{i}$ is chosen independently of the settings of the other bits.

The p-concept class of interest is the class of real-valued read-once formulas over the basis $\left\{\right.$ MUL, $\left.\operatorname{LIN}_{z ;} w_{1}, \ldots, w_{k}\right\}$ where MUL denotes ordinary multiplication of two or more real numbers, and $\operatorname{LIN}_{z ; w_{1}, \ldots, w_{k}}$ is the $k$-ary operator

$$
\operatorname{LIN}_{z ; w_{1}, \ldots, w_{k}}\left(y_{1}, \ldots, y_{k}\right)=z+\sum_{i=1}^{k} w_{i} y_{i}
$$

Here, $k$ may be any positive integer, and $z$ and the $w_{i}$ 's may be any real numbers which yield a function $\operatorname{LIN}_{z ; w_{1}, \ldots, w_{k}}$ having range $[0,1]$ on the domain $[0,1]^{k}$ (or, equivalently, on the domain $\left.\{0,1\}^{k}\right)$. We call formulas over this basis real formulas. For instance,

$$
\begin{aligned}
\operatorname{LIN}_{.4 ; 3 . .2}\left(\operatorname { M U L } \left(\operatorname{LIN}_{.5 ; .1,-.4}(\right.\right. & \left.\left.\left.x_{1}, x_{2}\right), \operatorname{LIN}_{1 ;-1}\left(x_{3}\right)\right), x_{4}\right)= \\
.4 & +.3 \cdot\left(.5+.1 x_{1}-.4 x_{2}\right) \cdot\left(1-x_{3}\right)+.2 x_{4}
\end{aligned}
$$

is a read-once real formula. Such formulas may also contain constants in the range $[0,1]$.

An easy induction argument shows that real formulas have range $[0,1]$, and so are p-concepts. Also, note that for Boolean-valued inputs, MUL is equivalent to AND, and LIN $_{1 ;-1}$ is equivalent to NOT. Thus, the class of read-once real formulas includes the class of read-once Boolean formulas over the basis \{AND, NOT\}, or, equivalently, $\{\mathrm{AND}, \mathrm{OR}, \mathrm{NOT}\}$.

Further, our criterion (1) for learning of real formulas implies the usual PAC learnability of Boolean formulas: if $c$ is a deterministic concept (i.e., a p-concept with range in $\{0,1\})$, and $h$ is a real-valued hypothesis satisfying (1), then $h^{\prime}=$ round $(h)=\lfloor h+1 / 2\rfloor$ is a $2 \epsilon$-good hypothesis for $c$ since

$$
\operatorname{Pr}_{x \in D}\left[h^{\prime}(x) \neq c(x)\right] \leq \operatorname{Pr}_{x \in D}[|h(x)-c(x)| \geq 1 / 2]
$$

and, by Markov's inequality,

$$
\epsilon \geq \mathbf{E}_{x \in D}[|h(x)-c(x)|] \geq \frac{1}{2} \mathbf{P r}_{x \in D}[|h(x)-c(x)| \geq 1 / 2] .
$$


Note that the class of read-once real formulas also includes the class of Boolean formulas which have been corrupted with a kind of random misclassification noise in which a "true" output of 0 or 1 is misclassified with probability $\eta_{0}$ or $\eta_{1}$, respectively. This is because such noise can be simulated by a single, output-level gate $\operatorname{LIN}_{\eta_{0}, 1-\eta_{0}-\eta_{1}}$ : on input 0 , this gate outputs $\eta_{0}$, and on input 1 , it outputs $1-\eta_{1}$. Thus, $\eta_{0}$ and $\eta_{1}$ are the respective probabilities that an input of 0 or 1 is "misclassified" or flipped by this gate. (This noise model is considered by Goldman, Kearns and Schapire (1990), and is a slight generalization of the noise model considered by Sloan (1988) and Angluin and Laird (1988).)

Thus, our result can be viewed as a demonstration of the learnability of readonce Boolean formulas with large doses of noise sprinkled throughout the formulas. Such noise may affect the formula's output ("misclassification noise"), the inputs ("attribute noise") or it might affect the output of every gate of the formula.

In general, we can regard a gate $\operatorname{LIN}_{z ; w_{1}, \ldots, w_{k}}$ as describing the behavior of a "noisy" or randomized Boolean gate as described in Section 1. If the $i$ th input is 1 with probability $p_{i}$, then the gate outputs 1 with probability

$$
z+\sum_{i=1}^{k} w_{i} p_{i}
$$

Thus, the probabilistic behavior of a formula constructed with such randomized gates is described by the p-concept obtained by replacing each randomized gate by a LIN gate, for appropriate choices of the LIN-gate parameters. (This can be proved rigorously, for instance, using a straightforward induction argument on the depth of the formula.)

\section{The learning algorithm}

Our learning procedure operates in three stages. In Stage I, we estimate the covariance of the formula $f$ with each variable $x_{i}$. This gives a very rough measure of $x_{i}$ 's correlation with $f$. Intuitively, we expect a highly correlated variable to give a lot of information about $f$, while a poorly correlated variable is likely to be essentially useless. This intuition turns out to be correct, and in Stage I, all variables with small covariances are identified, discarded and ignored in later stages. (Note, however, that the correctness of this intuition depends critically on the nature of both the formulas and the distributions we are considering.)

In Stage II, we use various statistical measures to construct an approximation of the target formula's topology or skeleton structure. By the skeleton of a real formula, we refer to the topological structure of the formula, including the type of each gate. In other words, the skeleton is the formula stripped of all its LIN-gate parameters. For instance, the formula in (2) has skeleton:

$$
\operatorname{LIN}\left(\operatorname{MUL}\left(\operatorname{LIN}\left(x_{1}, x_{2}\right), \operatorname{LIN}\left(x_{3}\right)\right), x_{4}\right) .
$$


Finally, in Stage III, we approximate the LIN-gate parameters for the skeleton inferred in Stage II, again using assorted statistical measures.

\subsection{Some preliminary facts}

Let $f$ be the target formula, and let $D$ be the target distribution. In what follows, all expectations are taken with respect to distribution $D$. For a function $g$, we also sometimes write $\bar{g}$ to denote its expectation:

$$
\bar{g}=\mathbf{E}[g]=\mathbf{E}_{x \in D}[g(x)] .
$$

We will be interested in the partial derivatives of $f$, which turn out to be central to our theory. We will be interested not only in partial derivatives with respect to variables $x_{i}$, but also with respect to subformulas $g$ of $f$. The following lemma demonstrates some useful properties of $\partial f / \partial g$.

Lemma 1 Let $g$ be a non-constant subformula of $f$ subsumed by some node $\lambda$. Then either $\partial f / \partial g$ or its negation $-\partial f / \partial g$ is a read-once real formula. Furthermore, no variable of $g$ is relevant to $\partial f / \partial g$.

Proof: Note that the lemma's statement is equivalent to the statements (1) that $\partial f / \partial g$ is either a nonnegative or a nonpositive function, and (2) that $|\partial f / \partial g|$ is a read-once real formula, none of whose variables are relevant to $g$.

We prove these statements by induction on the depth of $\lambda$ in $f$. If the depth is zero, then $f=g$, and $\partial f / \partial g=1$ so the lemma holds trivially.

Otherwise, suppose the output gate of $f$ is a gate $\operatorname{LIN}_{z ; w_{1}, \ldots, w_{k}}$. Then

$$
f=\operatorname{LIN}_{z ; w_{1}, \ldots, w_{k}}\left(f_{1}, \ldots, f_{k}\right)=z+w_{1} f_{1}+\cdots+w_{k} f_{k},
$$

for some subformulas $f_{1}, \ldots, f_{k}$. Since $\lambda$ has positive depth, it must occur in exactly one of these subformulas, say $f_{1}$. Then, by the chain rule,

$$
\frac{\partial f}{\partial g}=\frac{\partial f}{\partial f_{1}} \cdot \frac{\partial f_{1}}{\partial g}=w_{1} \cdot \frac{\partial f_{1}}{\partial g}
$$

By inductive hypothesis, $\partial f_{1} / \partial g$ is a nonpositive or a nonnegative function; thus, $\partial f / \partial g$ is as well. Since $\left|\partial f_{1} / \partial g\right|$ is also assumed to be a read-once real formula, and since $\left|w_{1}\right| \leq 1$ (by the properties of LIN gates), it must be that

$$
|\partial f / \partial g|=\operatorname{LIN}_{0 ;\left|w_{1}\right|}\left(\left|\partial f_{1} / \partial g\right|\right)
$$

is also a read-once real formula. Finally, if $x_{i}$ is relevant to $g$, then $x_{i}$ is not relevant to $\partial f / \partial g$ (since, by inductive hypothesis, it is not relevant to $\partial f_{1} / \partial g$ ). This proves the induction in this case.

Suppose now that the output gate of $f$ is a MUL gate that computes the product of $k \geq 2$ subformulas $f_{1}, \ldots, f_{k}$. Again, assume without loss of generality that $\lambda$ occurs in $f_{1}$. Applying the chain rule as before, we have that 


$$
\frac{\partial f}{\partial g}=\frac{\partial f}{\partial f_{1}} \cdot \frac{\partial f_{1}}{\partial g}=f_{2} \cdots f_{k} \cdot \frac{\partial f_{1}}{\partial g} .
$$

Since each of the subformulas $f_{i}$ is a read-once real formula, and by our inductive hypothesis, it follows as before that $\partial f / \partial g$ is either a nonpositive or a nonnegative function, and that

$$
\left|\frac{\partial f}{\partial g}\right|=\operatorname{MUL}\left(f_{2}, \ldots, f_{k},\left|\frac{\partial f_{1}}{\partial g}\right|\right)
$$

is a read-once real formula. If $x_{i}$ is relevant to $g$, then it does not occur in any of the subformulas $f_{2}, \ldots, f_{k}$, nor in $\partial f_{1} / \partial g$ (by inductive hypothesis); therefore, it is not relevant to $\partial f / \partial g$. Thus, the induction holds in this case as well. This proves the lemma.

In particular, this lemma shows that $|\partial f / \partial g|$ is always bounded by 1 .

Note that the formula $f$ could be "multiplied out" to give a polynomial over the variable set. This polynomial is linear in each variable $x_{i}$. This follows, for instance, from Lemma 1 which shows that $x_{i}$ is not relevant to $\partial f / \partial x_{i}$, and, therefore, that $\partial^{2} f / \partial x_{i}^{2}=0$. Thus, we can write

$$
f=u+v x_{i}
$$

for some functions $u$ and $v$ to which $x_{i}$ is not relevant. We call $u$ and $v$ the decomposition of $f$ in terms of $x_{i}$. In the same manner, $f$ can be decomposed in terms of any subformula $g$, and so can be written $f=u+v g$ for some functions $u$ and $v$ that do not contain any variable relevant to $g$.

Clearly, if $f=u+v x_{i}$, then

$$
\frac{\partial f}{\partial x_{i}}=v=\left(f \mid x_{i} \leftarrow 1\right)-\left(f \mid x_{i} \leftarrow 0\right) .
$$

Since, as proved above, $\partial f / \partial x_{i}$ is either nonpositive or nonnegative on all inputs,

$$
\left|\mathbf{E}\left[\partial f / \partial x_{i}\right]\right|=\mathbf{E}\left[\left|\partial f / \partial x_{i}\right|\right]=\mathbf{E}\left[\left|\left(f \mid x_{i} \leftarrow 1\right)-\left(f \mid x_{i} \leftarrow 0\right)\right|\right] .
$$

This latter expression is a natural measure of the influence of $x_{i}$, the degree to which $x_{i}$ 's value affects the value of $f$.

Henceforth, we let $B_{i}=\partial f / \partial x_{i}$.

In addition to the partial derivatives of $f$, we will also be interested in various central moments of $f$. For instance, for each variable $x_{i}$, we will be interested in the covariance $C_{i}$ of $f$ and $x_{i}$ :

$$
C_{i}=\mathbf{E}\left[(f-\bar{f})\left(x_{i}-\bar{x}_{i}\right)\right] .
$$

Speaking very loosely, $C_{i}$ measures the degree to which $f$ 's behavior is correlated with $x_{i}$. We will later see that variables that are weakly correlated with $f$ (as 
measured by the covariance) can be safely ignored by the learning algorithm without introducing significant error.

It is well known, and easily verified that

$$
C_{i}=\mathbf{E}\left[f x_{i}\right]-\mathbf{E}[f] \cdot \mathbf{E}\left[x_{i}\right] .
$$

Thus, if $f=u+v x_{i}$ as above, then

$$
\begin{aligned}
C_{i} & =\mathbf{E}\left[x_{i} \cdot\left(u+v x_{i}\right)\right]-\mathbf{E}\left[x_{i}\right] \cdot \mathbf{E}\left[u+v x_{i}\right] \\
& =\bar{x}_{i} \cdot \bar{u}+\bar{v} \cdot \mathbf{E}\left[x_{i}^{2}\right]-\bar{x}_{i} \cdot \bar{u}-\bar{v} \cdot \bar{x}_{i}^{2} \\
& =\bar{v} \bar{x}_{i}\left(1-\bar{x}_{i}\right) \\
& =\bar{B}_{i} \bar{x}_{i}\left(1-\bar{x}_{i}\right)
\end{aligned}
$$

where we have used our assumption of independence among variables, and the fact that $x_{i}^{2}=x_{i}$ since $x_{i}$ is Boolean. Thus, $C_{i}$ is "small" if $x_{i}$ is "sticky" (i.e., is almost always 0 or 1 ), or if $x_{i}$ has low influence.

Note that, by equation (4), $C_{i}$ can be well approximated by empirically estimating $\bar{x}_{i}, \bar{f}$ and $\mathbf{E}\left[f x_{i}\right]$. For instance, $\bar{x}_{i}$ is just the probability that $x_{i}=1$; similarly, $\mathbf{E}\left[f x_{i}\right]$ is the probability that an example is chosen that is labeled 1 , and for which $x_{i}=1$.

In Stages II and III, we will also be interested in the trivariance $T_{i j}$ of $f$ and distinct variables $x_{i}$ and $x_{j}$. This is defined to be

$$
T_{i j}=\mathbf{E}\left[(f-\bar{f})\left(x_{i}-\bar{x}_{i}\right)\left(x_{j}-\bar{x}_{j}\right)\right] .
$$

We will see that this value is quite important to our algorithm. For example, the type (MUL or LIN) of gate $\Gamma_{i j}$ can be determined from $T_{i j}$.

By multiplying out the product inside the expectations and using the independence of $x_{i}$ and $x_{j}$, it is straightforward to verify that

$$
T_{i j}=\mathbf{E}\left[f x_{i} x_{j}\right]-\mathbf{E}\left[f x_{i}\right] \cdot \mathbf{E}\left[x_{j}\right]-\mathbf{E}\left[f x_{j}\right] \cdot \mathbf{E}\left[x_{i}\right]+\mathbf{E}[f] \cdot \mathbf{E}\left[x_{i}\right] \cdot \mathbf{E}\left[x_{j}\right] .
$$

Thus, as was so for $C_{i}, T_{i j}$ can be well approximated from empirical estimates of $\bar{x}_{i}, \bar{x}_{j}, \bar{f}, \mathbf{E}\left[f x_{i}\right], \mathbf{E}\left[f x_{j}\right]$ and $\mathbf{E}\left[f x_{i} x_{j}\right]$.

Finally, just as $C_{i}$ turned out to ive related to the first partial derivative of $f$, so $T_{i j}$ is related to the second partial derivative. Since $f$ is linear in every variable, $f$ can be written as

$$
f=u_{0}+u_{1} x_{i}+u_{2} x_{j}+u_{3} x_{i} x_{j}
$$

for some functions $u_{0}, u_{1}, u_{2}$ and $u_{3}$ to which $x_{i}$ and $x_{j}$ are irrelevant. Clearly

$$
u_{3}=\frac{\partial^{2} f}{\partial x_{i} \partial x_{j}}
$$

which we henceforth denote $A_{i j}$. 
By an argument similar to that given above, it can be verified using equations (6), (7) and (8) that

$$
T_{i j}=\bar{A}_{i j} \bar{x}_{i}\left(1-\bar{x}_{i}\right) \bar{x}_{j}\left(1-\bar{x}_{j}\right) .
$$

This relationship will be very useful in what follows.

In Lemma 1 , we proved that either $\partial f / \partial x_{i}$ or its negation is a read-once real formula. Thus, in either case, the lemma implies that the second partial derivative $\partial^{2} f / \partial x_{i} \partial x_{j}$ has the same property - either it, or its negation, is a read-once real formula.

\subsection{Stage I: eliminating uncorrelated variables}

As described above, in Stage I, uncorrelated variables are eliminated. Our algorithm begins by estimating the expected values of $f, x_{i}, f x_{i}$ and $f x_{i} x_{j}$ for all variables $x_{i}$ and $x_{j}$. Applying Chernoff (1963) bounds, we see that a polynomial-size sample suffices to obtain estimates that, with probability at least $1-\delta$, are each within

$$
\tau=\frac{\epsilon_{0} \epsilon^{3}}{(20 n)^{5}}
$$

of the true expectation, where $\epsilon_{0}=\min \{\epsilon, 1 / n\}$. That is, for each of the random variables $R$ listed above, we require that

$$
|\mathbf{E}[R]-\hat{\mathbf{E}}[R]| \leq \tau,
$$

where $\hat{\mathbf{E}}[R]$ denotes the empirical mean of $R$ derived from the random sample. Henceforth, we assume that all of the random variables given above have the desired accuracy. As just mentioned, this will be the case with probability at least $1-\delta$.

In turn, we use these estimates in Stage I to obtain estimates $\hat{C}_{i}$ of the covariance $C_{i}$ of $f$ and $x_{i}$. In particular, we let

$$
\hat{C}_{i}=\hat{\mathbf{E}}\left[f x_{i}\right]-\hat{\mathbf{E}}[f] \cdot \hat{\mathbf{E}}\left[x_{i}\right] .
$$

Then we have

$$
\begin{aligned}
\mid C_{i} & -\hat{C}_{i} \mid \\
& =\left|\mathbf{E}\left[f x_{i}\right]-\mathbf{E}[f] \cdot \mathbf{E}\left[x_{i}\right]-\hat{\mathbf{E}}\left[f x_{i}\right]+\hat{\mathbf{E}}[f] \cdot \hat{\mathbf{E}}\left[x_{i}\right]\right| \\
& \leq\left|\mathbf{E}\left[f x_{i}\right]-\hat{\mathbf{E}}\left[f x_{i}\right]\right|+\hat{\mathbf{E}}[f] \cdot\left|\mathbf{E}\left[x_{i}\right]-\hat{\mathbf{E}}\left[x_{i}\right]\right|+\mathbf{E}\left[x_{i}\right] \cdot|\mathbf{E}[f]-\hat{\mathbf{E}}[f]| \\
& \leq 3 \tau .
\end{aligned}
$$

Let $\beta=\epsilon / 5 n$. If $\left|\hat{C}_{i}\right| \geq \beta+3 \tau$, we say that $x_{i}$ is correlated; in this case, $\left|C_{i}\right| \geq \beta$. In later stages, uncorrelated variables are ignored; for these variables, $\left|C_{i}\right| \leq \beta+6 \tau \leq \epsilon / 4 n$. 
We show next that uncorrelated variables can be ignored without introducing significant error. Let $f^{\prime}$ be the read-once real formula obtained from $f$ by replacing some variable $x_{i}$ by the constant $p_{i}=\bar{x}_{i}$. Then formula $f^{\prime}$ is just the p-concept obtained by regarding $x_{i}$ as a hidden variable - if we ignore $x_{i}$ 's value, then $f^{\prime}$ describes the probability that an assignment to the other variables is labeled 1 . Let $u, v$ be the decomposition of $f$ in terms of $x_{i}$ so that $f=u+v x_{i}$. Then $f^{\prime}=u+v p_{i}$, and so

$$
f-f^{\prime}=v\left(x_{i}-p_{i}\right)=\left(\partial f / \partial x_{i}\right)\left(x_{i}-p_{i}\right) .
$$

Thus, by independence and equation (5),

$$
\mathbf{E}\left[\left|f-f^{\prime}\right|\right]=\mathbf{E}\left[\left|\partial f / \partial x_{i}\right|\right] \cdot \mathbf{E}\left[\mid x_{i}-p_{i} \|\right]=\left|\bar{B}_{i}\right| \cdot 2 p_{i}\left(1-p_{i}\right)=2\left|C_{i}\right| .
$$

Note that if $x_{i}$ is uncorrelated, then this latter expression is at most $\epsilon / 2 n$.

Suppose $x_{1}, \ldots, x_{s}$ are the uncorrelated variables of $f$. Let $f_{0}=f$, let $f_{i}$ be obtained from $f_{i-1}$ by replacing $x_{i}$ with the constant $p_{i}$ for $1 \leq i \leq s$, and let $f_{\mathrm{I}}=f_{s}$. Then

$$
\mathbf{E}\left[\mid f-f_{\mathrm{I}} \| \leq \sum_{i=1}^{s} \mathbf{E}\left[\mid f_{i}-f_{i-1} \| \leq s \epsilon / 2 n \leq \epsilon / 2,\right.\right.
$$

since, by the preceding argument, $\mathbf{E}\left[\left|f_{i}-f_{i-1}\right|\right] \leq \epsilon / 2 n$.

Henceforth, we regard $f_{\mathrm{I}}$ as the target formula. Sampling according to $f_{\mathrm{I}}$ is achieved by simply ignoring the variables eliminated from $f$. In later stages, it is shown how to find a hypothesis $\hat{f}$ such that $\mathbf{E}\left[\left|\hat{f}-f_{\mathrm{I}}\right|\right] \leq \epsilon / 2$, and thus $\mathbf{E}[|\hat{f}-f|] \leq \epsilon$.

Note that we can easily handle at this point the special case that all the variables of $f$ are eliminated. For this case, $f_{\mathrm{I}}$ simply computes some constant function $p$. Since $\vec{f}=\vec{f}_{1}=p$, we simply let $\hat{f}=\hat{\mathbf{E}}[f]$, our previously obtained estimate of $\bar{f}$. Then $\mathbf{E}\left[\left|\hat{f}-f_{\mathrm{I}}\right|\right]=|\hat{\mathbf{E}}[f]-\mathbf{E}[f]| \leq r \leq \epsilon / 2$, and thus $\mathbf{E}[|f-\hat{f}|] \leq \epsilon$ as desired.

\subsection{Stage II: inferring the formula's skeleton}

Based on the results of Stage I, we can assume henceforth that none of the variables of $f$ are uncorrelated. Thus, in this and all subsequent sections, $f$ actually refers to the formula $f_{\mathrm{I}}$ of the previous section, and all of the variables discussed are assumed to be correlated.

We show in this section how a good approximation of the skeletal structure of $f$ can be obtained. Our approximation $\sigma^{\prime}$ will be sufficiently similar to the true skeleton $\sigma$ that the differences between the two structures will be provably inconsequential.

Note that the functional composition of two LIN gates is a LIN gate, and that $\operatorname{LIN}_{0 ; 1}$ is the identity function. Note also that any constant-valued subformulas 
occurring in $f$ can be eliminated by "absorbing" them into the rest of the formula, and that a single $k$-ary MUL gate can be replaced by a tree of binary MUL gates. These observations allow us to assume without loss of generality that no subformula of $f$ (including $f$ itself) is equal to a constant, and that the gates of $f$ occur in alternating layers of LIN and binary MUL gates, the output gate being a LIN gate, and every variable an input to a LIN gate. Thus, the topology or skeleton of $f$ is entirely determined first by the type of each gate, and second by the tree structure of $f$ with respect to its non-unary gates. Therefore, in reconstructing $f$ 's skeleton, we will be interested first in determining the type of each gate, and second in determining which gates are fed by which other gates, and in particular, which of two non-unary gates occurs deeper in the formula.

Our algorithm uses two tests for determining which of two gates is deeper. After describing the two tests, as well as a method for identifying the type of each gate, we show how the gathered information can be combined to construct an approximate skeleton.

For any three variables $x_{i}, x_{j}$ and $x_{k}$, we must have either that $\Gamma_{i j}=\Gamma_{i k}=\Gamma_{j k}$, or that exactly two of the gates $\Gamma_{i j}, \Gamma_{i k}$ and $\Gamma_{j k}$ are the same, and that the remaining gate is the deepest of the three. For instance, if $\Gamma_{i j}$ is the deepest gate, then it must feed $\Gamma_{i k}=\Gamma_{j k}$. Our purpose then is to determine which of these gates is deepest. This will be determined using the statistical measures described above.

Recall that good estimates were made in Stage I of the $C_{i}$ 's using the empirical means of various random variables. In the same manner, we can use these empirical estimates and equation (6) to obtain an estimate $\hat{T}_{i j}$ of each $T_{i j}$. Specifically, we let

$$
\hat{T}_{i j}=\hat{\mathbf{E}}\left[f x_{i} x_{j}\right]-\hat{\mathbf{E}}\left[f x_{i}\right] \cdot \hat{\mathbf{E}}\left[x_{j}\right]-\hat{\mathbf{E}}\left[f x_{j}\right] \cdot \hat{\mathbf{E}}\left[x_{i}\right]+\hat{\mathbf{E}}[f] \cdot \hat{\mathbf{E}}\left[x_{i}\right] \cdot \hat{\mathbf{E}}\left[x_{j}\right] .
$$

Then, by an argument similar to that used in proving the accuracy of $\hat{C}_{i}$, we have that

$$
\left|\hat{T}_{i j}-T_{i j}\right| \leq 8 \tau
$$

It will also be convenient to assume that each $\hat{C}_{i}$ and each $\hat{T}_{i j}$ is in the range $[-1,1]$; since $C_{i}$ and $T_{i j}$ are known to be in this range, we make this assumption without loss of generality. ("Clamping" estimates in this range can only improve their accuracy.)

\subsubsection{Determining gate types}

Our first goal in constructing the skeleton of $f$ is to determine the type of each gate. Specifically, for each pair of variables $x_{i}$ and $x_{j}$, we would like to determine if $\Gamma_{i j}$ is a LIN gate or a MUL gate. It turns out that this can be determined from our estimate of $T_{i j}$, as is shown in the following lemma.

Lemma 2 Let $x_{i}$ and $x_{j}$ be distinct variables. Then the following hold: 
1. If $\Gamma_{i j}$ is a LIN gate, then $T_{i j}=0$.

2. If $\Gamma_{i j}$ is a MUL gate, then $\left|T_{i j}\right| \geq\left|C_{i}\right| \cdot\left|C_{j}\right|$.

Proof: Suppose first that $\Gamma_{i j}$ is a gate $\operatorname{LIN}_{z ; w_{1}, \ldots, w_{k}}$. Then we can write $g_{i j}$ as the weighted sum of its inputs $g_{1}, \ldots, g_{k}$ :

$$
g_{i j}=z+w_{1} g_{1}+\cdots+w_{k} g_{k} .
$$

Without loss of generality, assume that $x_{i}$ and $x_{j}$ are relevant to $g_{1}$ and $g_{2}$, respectively. Then,

$$
\begin{aligned}
A_{i j} & =\frac{\partial^{2} f}{\partial x_{i} \partial x_{j}} \\
& =\frac{\partial}{\partial x_{i}}\left(\frac{\partial f}{\partial g_{i j}} \cdot \frac{\partial g_{i j}}{\partial g_{2}} \cdot \frac{\partial g_{2}}{\partial x_{j}}\right) \\
& =\frac{\partial}{\partial x_{i}}\left(\frac{\partial f}{\partial g_{i j}} \cdot w_{2} \cdot \frac{\partial g_{2}}{\partial x_{j}}\right)=0
\end{aligned}
$$

since $x_{i}$ is relevant to neither $\partial f / \partial g_{i j}$ nor $g_{2}$. Part 1 then follows from equation (9).

Suppose now that $\Gamma_{i j}$ is a MUL gate. We write subformula $g_{i j}$ as $g_{1} g_{2}$, the product of its inputs. (Recall that $\Gamma_{i j}$ is binary by assumption.) Suppose without loss of generality that $x_{i}$ and $x_{j}$ are relevant to $g_{1}$ and $g_{2}$, respectively. Then we have by the chain rule that

$$
\begin{aligned}
B_{i} & =\frac{\partial f}{\partial x_{i}} \\
& =\frac{\partial f}{\partial g_{i j}} \cdot \frac{\partial g_{i j}}{\partial g_{1}} \cdot \frac{\partial g_{1}}{\partial x_{i}} \\
& =\frac{\partial f}{\partial g_{i j}} \cdot \frac{\partial g_{1}}{\partial x_{i}} \cdot g_{2},
\end{aligned}
$$

and similarly

$$
B_{j}=\frac{\partial f}{\partial g_{i j}} \cdot \frac{\partial g_{2}}{\partial x_{j}} \cdot g_{1}
$$

Thus,

$$
A_{i j}=\frac{\partial^{2} f}{\partial x_{i} \partial x_{j}}=\frac{\partial f}{\partial g_{i j}} \cdot \frac{\partial g_{1}}{\partial x_{i}} \cdot \frac{\partial g_{2}}{\partial x_{j}} .
$$

Together, these imply that $\left|A_{i j}\right| \geq\left|B_{i}\right|\left|B_{j}\right|$ since $\left|\partial f / \partial g_{i j}\right|, g_{1}$ and $g_{2}$ are real formulas with range $[0,1]$. Applying equations (5) and (9), this proves part 2 of the lemma. 
This lemma is easily applied to the problem of determining the type of $\Gamma_{i j}$ : If $\Gamma_{i j}$ is a LIN gate, then $T_{i j}=0$ and so $\left|\hat{T}_{i j}\right|$ cannot exceed $8 \tau$. Conversely, if $\Gamma_{i j}$ is a MUL gate, then the lemma implies that

$$
\left|T_{i j}\right| \geq\left|C_{i}\right|\left|C_{j}\right| \geq \beta^{2}
$$

since each variable is assumed to be correlated; thus, in this case, $\left|\hat{T}_{i j}\right| \geq \beta^{2}-8 \tau>$ $8 \tau$. Thus, the type of $\Gamma_{i j}$ can be exactly determined from the magnitude of $\hat{T}_{i j}$ (given the assumption made above that all estimates have the desired accuracy). Our algorithm uses this technique to quickly ascertain the type of every non-unary gate in the formula.

\subsubsection{Determining relative gate depth: the type test}

The gate-type information collected in the preceding section is also helpful in determining the topology of the unknown formula. To be more precise, suppose that we would like to determine which of the gates $\Gamma_{i j}, \Gamma_{i k}$ or $\Gamma_{j k}$ occurs deepest in the formula. As noted above, at least two of these gates must be the same gate, and therefore must obviously be of the same type. The third gate may or may not be of the same type. If it is of a different type, then our algorithm will detect this difference and so will be able to conclude correctly that the gate of the odd type must in fact be the deepest of the three gates. For instance, if we find that $\Gamma_{i k}$ and $\Gamma_{j k}$ are MUL gates, but that $\Gamma_{i j}$ is a LIN gate, then the only possibility is that $\Gamma_{i k}=\Gamma_{j k}$, and that $\Gamma_{i j}$ is the deepest of the three gates. Of course, if all three gates have the same type, then the type test yields no information on the relative depth of the three gates.

Thus, after determining the type of each gate, our algorithm tests, for each ordered triple $x_{i}, x_{j}$ and $x_{k}$, whether $\Gamma_{i j}$ is of a different type from $\Gamma_{i k}$ and $\Gamma_{j k}$. If it finds that this is the case, then the algorithm can correctly conclude that $\Gamma_{i k}=\Gamma_{j k}$, and therefore $\Gamma_{i j}$ occurs deeper than this gate.

The results of all these type tests are organized in a directed graph $G_{0}$. The vertices of $G_{0}$ are unordered pairs $\{i, j\}$, for $i \neq j$. For each ordered triple of distinct indices $i, j, k$, our algorithm tests if $\Gamma_{i j}$ has a different type from $\Gamma_{i k}$ and $\Gamma_{j k}$. If this is the case, an edge is directed in $G_{0}$ from $\{i, j\}$ to $\{i, k\}$. Thus, as argued above, an edge is added to $G_{0}$ in this fashion only if $\Gamma_{i j}$ is deeper in $f$ than $\Gamma_{i k}$. Moreover, by transitivity, a path in $G_{0}$ from $\{i, j\}$ to $\left\{i^{\prime}, j^{\prime}\right\}$ implies that $\Gamma_{i j}$ is deeper than $\Gamma_{i^{\prime} j^{\prime}}$.

As noted above, the type test is one sided in the sense that if $\Gamma_{i j}, \Gamma_{i k}$ and $\Gamma_{j k}$ all have the same type, then nothing can be concluded about the relative depth of the three gates. However, the next lemma gives conditions under which the type test and its graph $G_{0}$ are guaranteed to give useful depth information.

Lemma 3 Let $x_{i}, x_{j}$ and $x_{k}$ be distinct variables of $f$, and assume $\Gamma_{i j}$ is deeper in $f$ than $\Gamma_{i k}=\Gamma_{j k}$. Suppose that either of the following conditions holds: 
1. $\Gamma_{i j}$ and $\Gamma_{i k}$ are of different types; or

2. $\Gamma_{i j}$ and $\Gamma_{i k}$ are of the same type, but there exists a non-unary gate $\Gamma_{i \ell}$ of a different type on the path in $f$ from $\Gamma_{i j}$ to $\Gamma_{i k}$.

Then there exists a path in $G_{0}$ from $\{i, j\}$ to $\{i, k\}$.

Proof: That condition 1 implies an edge in $G_{0}$ from $\{i, j\}$ to $\{i, k\}$ follows immediately from the remarks made above. Applying this fact twice shows that condition 2 implies that $\langle\{i, j\},\{i, \ell\}\rangle$ and $\langle\{i, \ell\},\{i, k\}\rangle$ are both edges in $G_{0}$.

Thus, if $\Gamma_{i j}$ is deeper than $\Gamma_{i k}=\Gamma_{j k}$, then the type test will succeed in making this determination unless every non-unary gate on the path in $f$ from $\Gamma_{i j}$ to $\Gamma_{i k}$ is of the same type. If all of the gates on this path are LIN gates, then they can all be collapsed into a single LIN gate. In other words, since we assume that the gates of $f$ occur in alternating levels of LIN/MUL gates, this "path" must actually consist of the single LIN gate $\Gamma_{i j}=\Gamma_{i k}=\Gamma_{j k}$.

If, on the other hand, all the non-unary gates on this path are MUL gates, then we cannot make such a collapse since there may be unary LIN gates along the path. Thus, the problem that remains is that of determining which of two MUL gates is deeper, specifically, in the case that the path from one gate to the other includes only MUL gates and unary LIN gates. This problem is (almost) solved in the next section using the estimated values $\hat{C}_{i}$ and $\hat{T}_{i j}$, which have already proved useful.

\subsubsection{Determining relative gate depth: the quotient test}

The second test used for determining the relative depth of two MUL gates is called the quotient test, and it has a flavor similar to that of the type test. In this section, and later in Stage III, we will be interested in the quantity

$$
E_{i j}=\frac{C_{i} C_{j}}{T_{i j}} .
$$

We will see that if $\Gamma_{i j}$ occurs deeper than $\Gamma_{i k}=\Gamma_{j k}$ then $E_{i k}=E_{j k}$, but $E_{i j}$ will differ significantly from $E_{i k}$ in most cases (the exception will be dealt with below). Thus, the quantities $E_{i j}$ will give a second method for determining which of the three gates $\Gamma_{i j}, \Gamma_{i k}, \Gamma_{j k}$ is deepest in $f$.

The following lemma gives a useful characterization of the quantity $E_{i j}$.

Lemma 4 Let $x_{i}$ and $x_{j}$ be distinct variables which meet at a MUL gate. Then

$$
E_{i j}=\mathbf{E}\left[\frac{\partial f}{\partial g_{i j}}\right] \cdot \bar{g}_{i j}
$$

Proof: In the proof of Lemma 2, expressions (11), (12) and (13) were derived for $B_{i}, B_{j}$ and $A_{i j}$. From these, and equations (5) and (9), it follows that 


$$
E_{i j}=\frac{C_{i} C_{j}}{T_{i j}}=\frac{\bar{B}_{i} \bar{B}_{j}}{\bar{A}_{i j}}=\mathbf{E}\left[\frac{\partial f}{\partial g_{i j}}\right] \cdot \bar{g}_{1} \bar{g}_{2}
$$

Here, as in Lemma $2, g_{1}$ and $g_{2}$ are the inputs to $\Gamma_{i j}$. Since, by independence, $\bar{g}_{i j}=\bar{g}_{1} \bar{g}_{2}$, this proves the lemma.

Thus, if $\Gamma_{i k}=\Gamma_{j k}$, then Lemma 4 shows that $E_{i k}=E_{j k}$.

To estimate the quantities $E_{i j}$, we naturally use

$$
\hat{E}_{i j}=\frac{\hat{C}_{i} \hat{C}_{j}}{\hat{T}_{i j}}
$$

To show that this is a good estimate, we will need the following lemma on estimating quotients.

Lemma 5 For $b$ and $\hat{b}$ nonzero,

$$
\left|\frac{\hat{a}}{\hat{b}}-\frac{a}{b}\right| \leq \frac{|\hat{a}-a|}{|\hat{b}|}+\left|\frac{a}{b}\right| \cdot \frac{|\hat{b}-b|}{|\hat{b}|} \text {. }
$$

Proof:

$$
\left|\frac{\hat{a}}{\hat{b}}-\frac{a}{b}\right|=\left|\frac{\hat{a}}{\hat{b}}-\frac{a}{\hat{b}}+\frac{a}{\hat{b}}-\frac{a}{b}\right| \leq \frac{|\hat{a}-a|}{|\hat{b}|}+\left|\frac{a b-a \hat{b}}{b \hat{b}}\right|=\frac{|\hat{a}-a|}{|\hat{b}|}+\left|\frac{a}{b}\right| \cdot \frac{|\hat{b}-b|}{|\hat{b}|} .
$$

Let $\gamma=28 \tau / \beta^{2}$.

Lemma 6 For any two distinct variables $x_{i}$ and $x_{j},\left|E_{i j}-\hat{E}_{i j}\right| \leq \gamma$.

Proof: Applying Lemma 5, we have that

$$
\left|E_{i j}-\hat{E}_{i j}\right| \leq \frac{\left|C_{i} C_{j}-\hat{C}_{i} \hat{C}_{j}\right|}{\left|\hat{T}_{i j}\right|}+\left|E_{i j}\right| \cdot \frac{\left|T_{i j}-\hat{T}_{i j}\right|}{\left|\hat{T}_{i j}\right|}
$$

Since $\left|E_{i j}\right| \leq 1$ (by Lemmas 1 and 4 ), and since $\left|C_{i}\right|$ and $\left|\hat{C}_{j}\right|$ are also bounded by 1 , this is at most

$$
\frac{\left|C_{i}-\hat{C}_{i}\right|+\left|C_{j}-\hat{C}_{j}\right|+\left|T_{i j}-\hat{T}_{i j}\right|}{\left|\hat{T}_{i j}\right|} \leq \frac{14 \tau}{\beta^{2}-8 \tau} \leq \gamma
$$

as claimed. Here, we have used Lemma 2 to lower bound $\left|\hat{T}_{i j}\right|$. 
Thus, $\hat{E}_{i j}$ is a good estimate of $E_{i j}$, and in particular, if $\Gamma_{i k}=\Gamma_{j k}$ then $E_{i k}=E_{j k}$ and $\left|\hat{E}_{i k}-\hat{E}_{j k}\right| \leq 2 \gamma$. Therefore, for every ordered triple of variables $x_{i}, x_{j}$ and $x_{k}$, our algorithm tests if all of the following hold:

$$
\begin{aligned}
& \left|\hat{E}_{i k}-\hat{E}_{j k}\right| \leq 2 \gamma, \\
& \left|\hat{E}_{i j}-\hat{E}_{i k}\right|>2 \gamma \\
& \left|\hat{E}_{i j}-\hat{E}_{j k}\right|>2 \gamma .
\end{aligned}
$$

If all of these hold, then our algorithm can correctly conclude that $E_{i j} \neq E_{i k}=E_{j k}$ (since at least two of these values must be equal) and therefore that $\Gamma_{i j}$ is deeper than $\Gamma_{i k}=\Gamma_{j k}$.

Such information is added to the graph $G_{0}$ used for the type test. Specifically, an edge is added from $\{i, j\}$ to $\{i, k\}$ whenever the quotient test implies that $\Gamma_{i j}$ is deeper than $\Gamma_{i k}$.

As was the case for the type test, the quotient test is one sided: it may be that two or three of the differences $\left|\hat{E}_{i k}-\hat{E}_{j k}\right|,\left|\hat{E}_{i j}-\hat{E}_{i k}\right|$ and $\left|\hat{E}_{i j}-\hat{E}_{j k}\right|$ do not exceed $2 \gamma$. In this case, the quotient test fails to give any useful information about the relative depths of $\Gamma_{i j}, \Gamma_{i k}$ and $\Gamma_{j k}$. However, this can only happen if $\hat{E}_{i j}, \hat{E}_{i k}$ and $\hat{E}_{j k}$ are so close that the difference between any two of these estimates is bounded by $4 \gamma$. This fact will help us later in handling the situation that neither the type nor the quotient test succeeds in discerning the relative depth of two gates.

\subsubsection{Constructing the skeleton from $G_{0}$}

Finally, we are ready to show how an "approximate" skeleton of $f$ can be computed from the graph $G_{0}$. First, to reiterate what was pointed out above, an edge in $G_{0}$ from $\{i, j\}$ to $\{i, k\}$ indicates that $\Gamma_{i j}$ must be deeper than $\Gamma_{i k}$ (given our assumption that all estimates are good); however, if $\Gamma_{i j}$ is deeper than $\Gamma_{i k}$, there need not be a corresponding edge in $G_{0}$ (i.e., both tests above may have failed).

We can construct another graph $G_{1}$ from $G_{0}$ with a slightly different property: Graph $G_{1}$ has the same vertex set as $G_{0}$, and is obtained from $G_{0}$ by adding all edges from $\{i, j\}$ to $\{i, k\}, j \neq k$ which do not "contradict" $G_{0}$, i.e., for which there does not exist a path in $G_{0}$ from $\{i, k\}$ to $\{i, j\}$.

Thus, if $\Gamma_{i j}$ is deeper than $\Gamma_{i k}$, or if $\Gamma_{i j}=\Gamma_{i k}$ then $G_{1}$ must contain an edge from $\{i, j\}$ to $\{i, k\}$ since in this case, $G_{0}$ certainly will not contain a path in the opposite direction. Furthermore, if there exists a path in $G_{1}$ from $\{i, j\}$ to $\{k, \ell\}$, but no path in the reverse direction, then we can conclude that $\Gamma_{i j}$ is deeper in $f$ than $\Gamma_{k \ell}$. When there exist paths connecting these vertices in both directions - that is, when $\{i, j\}$ and $\{k, \ell\}$ are in the same strongly connected component of $G_{1}-$ 
there is no information about which gate is deeper. In this case, we collapse all of the gates corresponding to vertices of this strongly connected component (including $\Gamma_{i j}$ and $\Gamma_{k \ell}$ ). In the remainder of this section, we give an algorithm for forming a skeleton for $f$ by collapsing gates in this manner and we show that the skeleton we obtain is a good approximation of the true skeleton.

We say two vertices of $G_{1}$ are equivalent (with respect to $G_{1}$ ) if they are in the same strongly connected component. There are two ways in which vertices $\{i, j\}$ and $\{k, \ell\}$ may be equivalent. First, they may be equivalent if $\Gamma_{i j}=\Gamma_{k \ell}$, in which case $\Gamma_{i j}$ and $\Gamma_{k \ell}$ can and certainly should be collapsed into the same gate. Alternatively, as suggested above, $\{i, j\}$ and $\{k, \ell\}$ may be equivalent due to insufficient information regarding the relative depth of $\Gamma_{i j}$ and $\Gamma_{k \ell}$. Note that by the results of Section 3.3.2, this can only be a problem for MUL gates which are separated by unary LIN gates.

We say that a LIN gate of $f$ is trapped if it is immediately fed by some MUL gate $\Gamma_{i j}$, and it immediately feeds another MUL gate $\Gamma_{k \ell}$ for some indices $i, j, k, \ell$ for which $\{i, j\}$ and $\{k, \ell\}$ are equivalent. In other words, the LIN gate is trapped if it is "surrounded" above and below by gates whose indices are in the same strongly connected component of $G_{1}$. Trapped LIN gates represent holes in our algorithm's knowledge of the formula's structure. Fortunately, it turns out that these gates have so little effect on the formula's behavior that they can be safely ignored. Ignoring trapped LIN gates essentially is equivalent to collapsing the surrounding MUL gates into a single (multi-input) MUL gate.

Thus, as will be shown below, the strongly connected components of $G_{1}$ generally correspond to connected regions of $f$ (where we view $f$ as a graph whose vertices are the gates, and whose edges are the "wires" connecting the gates), and that these connected regions can be safely approximated by simple LIN or MUL gates.

Let $\sigma$ denote the true skeleton of $f$. Recall that we have assumed without loss of generality that the MUL gates of $\sigma$ are all binary, and that the gates of $f$ occur in alternating layers of LIN/MUL gates, the output gate being a LIN gate, and every input variable immediately feeding a LIN gate.

Let $\sigma^{\prime}$ be another skeleton derived from $\sigma$ by first deleting all trapped LIN gates, and by then collapsing now adjacent MUL gates. That is, the deletion of a trapped LIN gate necessarily results in one MUL gate immediately feeding another; in constructing $\sigma^{\prime}$, we collapse these two gates into a single, multi-input Mul gate, purely for the sake of convenience and uniformity.

Clearly, as was so for $\sigma$, the gates of $\sigma^{\prime}$ occur in alternating layers of MUL and LIN gates with LIN gates occurring at the output and input levels. However, unlike $\sigma$, MUL gates in $\sigma^{\prime}$ may have more than two inputs.

We let $\Gamma_{i j}^{\prime}$ denote that gate in $\sigma^{\prime}$ at which variables $x_{i}$ and $x_{j}$ meet. To formalize the transformation of $\sigma$ into $\sigma^{\prime}$, we let $\rho(\Gamma)$ denote that node $\Gamma^{\prime}$ of $\sigma^{\prime}$ into which node $\Gamma$ of $\sigma$ is transformed. Specifically, it can be verified that $\rho$ has the following properties:

- For non-unary gates $\Gamma_{i j}$ of $\sigma, \rho\left(\Gamma_{i j}\right)=\Gamma_{i j}^{\prime}$. 
- All variables are mapped to themselves: $\rho\left(x_{i}\right)=x_{i}$.

- If $\Gamma$ is a non-trapped unary LIN gate, then $\rho(\Gamma)$ is that gate immediately fed by $\rho(\lambda)$, where $\lambda$ immediately feeds $\Gamma$.

- Finally, if $\Gamma$ is a trapped LIN gate, then $\Gamma$ is actually eliminated in the construction of $\sigma^{\prime}$; nevertheless, we define $\rho(\Gamma)$ to be $\rho(\lambda)$ where $\lambda$ is the MuL gate that immediately feeds $\Gamma$ (or, equivalently, we could have used the MUL gate immediately fed by $\Gamma$ ).

Clearly, the function $\rho$ maps onto the set of all nodes of $\sigma^{\prime}$. Furthermore, $\rho$ is one-to-one if its domain is restricted to non-trapped LIN gates and variables.

Let

$$
\rho^{-1}\left(\Gamma^{\prime}\right)=\left\{\Gamma: \rho(\Gamma)=\Gamma^{\prime}\right\}
$$

be the set of $\sigma$-nodes mapped by $\rho$ to $\Gamma^{\prime}$. Note that, by construction of $\sigma^{\prime}$, every set $\rho^{-1}\left(\Gamma^{\prime}\right)$ is connected; that is, if $\lambda_{1}$ and $\lambda_{2}$ are both contained in this set, then all of the nodes along the unique path in $f$ from $\lambda_{1}$ to $\lambda_{2}$ are also contained in the set. Thus, every set $\rho^{-1}\left(\Gamma^{\prime}\right)$ has a unique member of minimal depth. We call this minimal-depth node $\alpha\left(\Gamma^{\prime}\right)$, and we say that a $\sigma$-node $\Gamma$ is an apex node if it is in the image of $\alpha$.

We show next how the skeleton $\sigma^{\prime}$ can be constructed from $G_{1}$. Later, in Stage III, we will show that $\sigma^{\prime}$ can be used to construct a good approximation of $f$.

Figure 1 shows our algorithm for constructing $\sigma^{\prime}$ from $G_{1}$. The algorithm maintains a family of skeletons $F$. On each iteration of the loop, several of these skeletons are combined into one. Taking strongly connected components with no incoming edges at line 4 guarantees that the algorithm will start from the inputs and work bottom-up. We will show that only one skeleton remains in $F$ upon termination.

The operator COND-LIN $(s)$ used at lines 10 and 14 returns $s$ if the output gate of $s$ is a LIN gate, and LIN $(s)$ otherwise. (In other words, a LIN gate is added to $s$, but only if necessary.) Also, the set $\operatorname{rel}(s)$ for skeleton $s$ is the set of variables relevant to $s$. Finally, $G-H$ at line 12 denotes the graph obtained from $G$ by deleting all vertices in $H$, and any edges incident to these deleted vertices.

We say a condition holds at all times if it holds between each iteration of the main loop.

The algorithm clearly halts since some vertex of $G$ is removed on each iteration.

Lemma 7 At all times, if $s_{1}$ and $s_{2}$ are distinct members of $F$, then $\operatorname{rel}\left(s_{1}\right) \cap$ $\operatorname{rel}\left(s_{2}\right)=\emptyset$. Also, $\bigcup_{s \in F} \operatorname{rel}(s)=\left\{x_{1}, \ldots, x_{n}\right\}$ (assuming all of the variables are correlated).

Proof: Let $s_{0}$ and $E$ be as in the algorithm. Then, using the fact that $\operatorname{rel}\left(s_{0}\right)=$ $\bigcup_{s \in E} \operatorname{rel}(s)$, the lemma follows by an easy induction on the number of iterations of the main loop. 
Input: the graph $G_{1}$, and the type of each gate of $f$

Output: a skeleton $\sigma^{\prime}$ that approximates the true skeleton $\sigma$ of $f$

Procedure:

$1 G \leftarrow G_{1}$

$2 F \leftarrow\left\{x_{i}: 1 \leq i \leq n\right\}$

3 repeat while $G$ is not empty

4 find a strongly connected component $H$ of $G$ with no incoming edges

$5 \quad E \leftarrow\left\{s \in F:\{i, j\} \in H\right.$ and $\left.x_{i} \in \operatorname{rel}(s)\right\}$

6 let $\{i, j\}$ be any node of $H$, and let $E=\left\{s_{1}, \ldots, s_{|E|}\right\}$

7 if $\Gamma_{i j}$ is a LIN gate then

$8 \quad s_{0} \leftarrow \operatorname{LIN}\left(s_{1}, \ldots, s_{|E|}\right)$

9 else $\left\{\Gamma_{i j}\right.$ is a MUL gate $\}$

$10 s_{0} \leftarrow \operatorname{MUL}\left(\operatorname{COND}-\operatorname{Lin}\left(s_{1}\right), \ldots, \operatorname{COND}-\operatorname{Lin}\left(s_{|E|}\right)\right)$

$11 \quad F \leftarrow(F-E) \cup\left\{s_{0}\right\}$

$12 G \leftarrow G-H$

13 end

14 output COND-LIN $(s)$, where $s$ is the only member of $F$

Figure 1. An algorithm for inferring a good skeleton from $G_{1}$.

Lemma 8 At all times, if $\{i, j\}$ is not a vertex of $G$ then $\left\{x_{i}, x_{j}\right\} \subset \operatorname{rel}(s)$ for some $s \in F$.

Proof: Note that $\{i, j\}$ is removed from $G$ at line 12 only if $x_{i}$ and $x_{j}$ are relevant to skeletons in $E$.

Lemma 9 Upon termination of the main loop, $|F|=1$.

Proof: If, upon termination, $F$ contained two skeletons $s_{1}$ and $s_{2}$, then each must have distinct relevant variables $x_{i}$ and $x_{j}$, respectively, by Lemma 7 . By Lemma 8 , this implies $\{i, j\} \in G$, contradicting the fact that $G$ is empty.

Lemma 10 At all times, if $s \in F$ then $s$ is a subskeleton of $\sigma^{\prime}$.

Proof: By induction on the number of iterations of the main loop. Initially, the lemma holds trivially.

Consider the state of the algorithm immediately before $G$ is modified at line 12 . Let $s_{0}$ and $E$ be as in the algorithm. Let $\{k, \ell\} \in H$ be such that $\Gamma_{k \ell}$ is a gate of minimal depth in the set $\left\{\Gamma_{i j}:\{i, j\} \in H\right\}$. (That is, $\Gamma_{k \ell}$ does not feed any gate in this set.)

Claim 1: $\operatorname{rel}\left(s_{0}\right)=\operatorname{rel}\left(g_{k \ell}\right)$.

Proof of Claim 1: Note first that, by definition of $E$,

$$
\operatorname{rel}\left(s_{0}\right)=\left\{x_{i}:\{i, j\} \in H\right\} .
$$


Thus, if $x_{i} \in \operatorname{rel}\left(s_{0}\right)$ then $\{i, j\} \in H$ for some $j$. Let $T$ be the set

$$
T=\left\{\left\{i^{\prime}, j^{\prime}\right\} \in G: \Gamma_{i^{\prime} j^{\prime}} \text { feeds } \Gamma_{k \ell} \text { or } \Gamma_{i^{\prime} j^{\prime}}=\Gamma_{k \ell}\right\} .
$$

We will show that $\{i, j\} \in T$; this implies that $x_{i}$ is in the subformula subsumed by $\Gamma_{k \ell}$, and thus $x_{i} \in \operatorname{rel}\left(g_{k \ell}\right)$.

If $\{i, j\} \notin T$, then since $\{k, \ell\} \in T$ and since $\{i, j\}$ and $\{k, \ell\}$ are equivalent, there must be a path in $H$ from $\{i, j\}$ to $\{k, \ell\}$, and there must be an edge on this path from some node $\left\{i^{\prime}, j^{\prime}\right\} \notin T$ to some other node $\left\{i^{\prime}, k^{\prime}\right\} \in T$. This means that $\Gamma_{i^{\prime} k^{\prime}}$ feeds or is equal to $\Gamma_{k \ell}$, but $\Gamma_{i^{\prime} j^{\prime}}$ is not in $g_{k \ell}$, the subformula subsumed by $\Gamma_{k \ell}$. In particular, this means that $\Gamma_{i^{\prime} k^{\prime}}$ feeds $\Gamma_{i^{\prime} j^{\prime}}$. Moreover, $\Gamma_{k \ell}$, being the root of $g_{k \ell}$, must certainly be on the path from $\Gamma_{i^{\prime} k^{\prime}}$ to $\Gamma_{i^{\prime} j^{\prime}}$. Thus, $\Gamma_{i^{\prime} k^{\prime}}$ feeds (or is equal to) $\Gamma_{k \ell}$, which in turn feeds $\Gamma_{i^{\prime} j^{\prime}}$. Therefore, there exist paths in $G_{1}$ from $\left\{i^{\prime}, k^{\prime}\right\}$ to $\{k, \ell\}$, and from $\{k, \ell\}$ to $\left\{i^{\prime}, j^{\prime}\right\}$. Since an edge is directed from $\left\{i^{\prime}, j^{\prime}\right\}$ to $\left\{i^{\prime}, k^{\prime}\right\}$, this implies that $\left\{i^{\prime}, j^{\prime}\right\}$ is equivalent to $\{k, \ell\}$, and so must be in $H$. However, this contradicts the definition of $\{k, \ell\}$ since $\{k, \ell\}$ is deeper than $\left\{i^{\prime}, j^{\prime}\right\}$.

Thus, $\operatorname{rel}\left(s_{0}\right) \subset \operatorname{rel}\left(g_{k \ell}\right)$.

Conversely, suppose $x_{i} \in \operatorname{rel}\left(g_{k \ell}\right)$. Then $\Gamma_{i \ell}$ feeds or is equal to $\Gamma_{k \ell}$. Thus, there is a path in $G_{1}$ from $\{i, \ell\}$ to $\{k, \ell\}$. Since $H$ is assumed to have no incoming edges, this implies that either $\{i, \ell\} \in H$ or $\{i, \ell\} \notin G$. If $\{i, \ell\} \notin G$, then by Lemma 8 , $x_{i} \in \operatorname{rel}\left(s_{0}\right)$ since $x_{\ell} \in \operatorname{rel}\left(s_{0}\right)$. If $\{i, \ell\} \in H$, then $x_{i} \in \operatorname{rel}\left(s_{0}\right)$ by construction. Thus, $\operatorname{rel}\left(s_{0}\right)=\operatorname{rel}\left(g_{k \ell}\right)$, proving Claim 1 .

Let $s^{\prime}$ be the subskeleton of $\sigma^{\prime}$ subsumed by gate $\Gamma_{k \ell}^{\prime}=\rho\left(\Gamma_{k \ell}\right)$. We will show that $s_{0}=s^{\prime}$, completing the induction and proving the lemma.

Claim 2: $\operatorname{rel}\left(s_{0}\right)=\operatorname{rel}\left(s^{\prime}\right)$.

Proof of Claim 2: We prove this claim by showing that $\operatorname{rel}\left(s^{\prime}\right)=\operatorname{rel}\left(g_{k \ell}\right)$. In fact, it suffices to show that $\Gamma_{k \ell}$ is an apex node, since $\operatorname{rel}\left(\lambda^{\prime}\right)=\operatorname{rel}\left(\alpha\left(\lambda^{\prime}\right)\right)$ for any $\sigma^{\prime}$-node $\lambda^{\prime}$.

First, if $\Gamma_{k \ell}$ is a LiN gate, then it must be an apex gate since it is the only member of $\rho^{-1}\left(\Gamma_{k \ell}^{\prime}\right)$.

Suppose then that $\Gamma_{k \ell}$ is a MUL gate, but not an apex node. Then $\Gamma_{k \ell}$ immediately feeds a trapped LIN gate, which in turn feeds some other MUL gate $\Gamma_{i j}$ for which $\{i, j\}$ and $\{k, \ell\}$ are equivalent. But then $\{i, j\} \in H$, contradicting the definition of $\Gamma_{k \ell}$.

This proves Claim 2.

By inductive hypothesis, each skeleton in $E$ is a subskeleton of $\sigma^{\prime}$. Claim 2 implies that each of these skeletons is in fact a subskeleton of $s^{\prime}$.

Suppose $x_{i}$ and $x_{j}$ are relevant variables, respectively, of two distinct skeletons in $E$. Then $\{i, j\} \in G$, by Lemmas 7 and 8 . Also, $\Gamma_{i j}$ must feed or equal $\Gamma_{k \ell}$ by Claim 1. Thus, there must be a path in $G$ from $\{i, j\}$ to $\{k, \ell\}$, and therefore, $\{i, j\}$ must be in $H$.

We claim that $\Gamma_{i j}$ and $\Gamma_{k \ell}$ must be of the same type. Suppose not. Then there must be a gate $\Gamma_{i j^{\prime}}$ that immediately feeds a gate $\Gamma_{i k^{\prime}}$ of a different type, and such that both gates are on the inclusive path from $\Gamma_{i j}$ to $\Gamma_{k \ell}$. Since there is a path in $H$ from $\{k, \ell\}$ to $\{i, j\}$, there must be some edge in $H$ directed from $\left\{i^{\prime}, k^{\prime \prime}\right\}$ to 
$\left\{i^{\prime}, j^{\prime \prime}\right\}$ where these indices are such that $\Gamma_{i j^{\prime}}$ feeds $\Gamma_{i^{\prime} k^{\prime \prime}}$, and $\Gamma_{i^{\prime} j^{\prime \prime}}$ feeds or equals $\Gamma_{i j^{\prime}}$. However, because there is a path in $f$ from $\Gamma_{i^{\prime} j^{\prime \prime}}$ to $\Gamma_{i^{\prime} k^{\prime \prime}}$ that passes through $\Gamma_{i j^{\prime}}$ and $\Gamma_{i k^{\prime}}$, and because these four gates cannot all be of the same type, we can conclude using Lemma 3 that there must be a path in $G_{0}$ from $\left\{i^{\prime}, j^{\prime \prime}\right\}$ to $\left\{i^{\prime}, k^{\prime \prime}\right\}$. But this contradicts the existence of the edge in $G_{1}$ from $\left\{i^{\prime}, k^{\prime \prime}\right\}$ to $\left\{i^{\prime}, j^{\prime \prime}\right\}$.

Therefore, $\Gamma_{i j}$ and $\Gamma_{k \ell}$ must be of the same type. This implies that $\Gamma_{i j}^{\prime}$ and $\Gamma_{k \ell}^{\prime}$ are also of the same type as $\Gamma_{k \ell}$. If $\Gamma_{k \ell}$ is a LIN gate, we have shown then that every gate on the path from $\Gamma_{i j}$ to $\Gamma_{k \ell}$ is a LIN gate, and thus $\Gamma_{i j}=\Gamma_{k \ell}$, which implies $\Gamma_{i j}^{\prime}=\Gamma_{k \ell}^{\prime}$.

If $\Gamma_{k \ell}$ is a MUL gate, we have shown that every non-unary gate on the path from $\Gamma_{i j}$ to $\Gamma_{k \ell}$ is a MUL gate, and furthermore, that every LIN gate on this path is trapped (since $\{i, j\}$ and $\{k, \ell\}$ are equivalent). Therefore, in this case as well, $\Gamma_{i j}^{\prime}=\Gamma_{k \ell}^{\prime}$.

This proves that $s^{\prime}=s_{0}$, as desired, and completes the induction.

Combining Lemmas 7, 9, and 10, it follows immediately that the algorithm of Figure 1 outputs $\sigma^{\prime}$. The algorithm clearly runs in polynomial time.

\subsection{Stage III: inferring the LIN-gate parameters}

In the final stage, our algorithm "fills in" the missing LIN-gate parameters for the skeleton inferred in Stage II.

The first problem we face is that $\sigma^{\prime}$ is only an approximation of the true skeleton $\sigma$, to which we have no direct access. Nevertheless, we will be able to show that the procedure we describe for constructing a complete formula from a skeleton yields the same result whether applied to the true skeleton $\sigma$ or the approximate skeleton $\sigma^{\prime}$. This fact will allow us to assume in proving the correctness of the procedure that we are actually dealing with the true skeleton $\sigma$.

Our algorithm computes the LIN-gate parameters of $f$ from the bottom up: the algorithm visits each node of the skeleton, starting with variables. No node is visited until all those that feed it have been visited. When some node $\lambda$ is visited which subsumes subformula $g$, we might like to compute a formula that approximates the function $g$; unfortunately, this does not seem to be possible because a given subformula may not be uniquely defined: by rearranging the LIN-gate parameters which occur in $f$, we can change the identity of individual subformulas (by constant factors) without altering the functional behavior of $f$. For instance, although the formulas

$$
\operatorname{LIN} .1 ;-.8\left(\operatorname{MUL}\left(\operatorname{LIN} .2 ; .1, .2\left(x_{1}, x_{2}\right), \operatorname{LIN}_{0 ; .3}\left(x_{3}\right)\right)\right.
$$

and

$$
\operatorname{LIN}_{.1 ;-.2}\left(\operatorname{MUL}\left(\operatorname{LIN}_{.4 ; .2,4}\left(x_{1}, x_{2}\right), \operatorname{LiN}_{0 ; .6}\left(x_{3}\right)\right)\right.
$$


are equal (viewed as functions), they clearly contain many pairs of corresponding subformulas which are not equal.

Instead then, our algorithm infers an approximation of a function closely related to $g$, namely,

$$
h=\mathbf{E}[\partial f / \partial g] \cdot g .
$$

We call $h$ the canonical form of $g$. Unlike $g$, the canonical form $h$ is uniquely determined. Note also that if $g$ is the entire formula $f$, then $h=g=f$.

Another nice property of the canonical form is that its expected value $\mathbf{E}[\partial f / \partial g] \cdot \vec{g}$ can be statistically approximated (given $f$ 's skeleton), as will be seen below. We write $\chi(\lambda)$ to denote this expectation. The next lemma yields a technique for estimating $\chi(\lambda)$, a key quantity used by our reconstruction procedure.

Recall from Section 3.3.3 that $E_{i j}$ is defined to be $C_{i} \cdot C_{j} / T_{i j}$.

Lemma 11 Let $\Gamma_{i j}$ be a MUL gate of $f$.

1. $\chi\left(\Gamma_{i j}\right)=E_{i j}$.

2. If $\lambda$ immediately feeds $\Gamma_{i j}$, then $\chi(\lambda)=\chi\left(\Gamma_{i j}\right)$.

3. For any variable $x_{i}, \chi\left(x_{i}\right)=C_{i} /\left(1-\bar{x}_{i}\right)$.

Proof: Part 1 is simply a restatement of Lemma 4.

Let $g$ be the subformula subsumed by $\lambda$. Then we can write $g_{i j}=g \cdot g^{\prime}$ for some subformula $g^{\prime}$. Then by independence and the chain rule,

$$
\begin{aligned}
\chi(\lambda) & =\mathbf{E}[\partial f / \partial g] \cdot \bar{g} \\
& \doteq \mathbf{E}\left[\partial f / \partial g_{i j}\right] \cdot \mathbf{E}\left[\partial g_{i j} / \partial g\right] \cdot \bar{g} \\
& =\mathbf{E}\left[\partial f / \partial g_{i j}\right] \cdot \bar{g}^{\prime} \bar{g}=\chi\left(\Gamma_{i j}\right) .
\end{aligned}
$$

Finally, by equation (5), $\chi\left(x_{i}\right)=\mathbf{E}\left[\partial f / \partial x_{i}\right] \cdot \bar{x}_{i}=C_{i} /\left(1-\bar{x}_{i}\right)$.

We use Lemma 11 to guide us in assigning an estimate $\hat{\chi}\left(\lambda^{\prime}\right)$ to every node of $\sigma^{\prime}$. Specifically, if $\lambda^{\prime}$ is a MUL gate, then we set $\hat{\chi}\left(\lambda^{\prime}\right)=\hat{E}_{k \ell}$ where $k, \ell$ is an arbitrarily chosen pair for which $\Gamma_{k \ell}^{\prime}=\lambda^{\prime}$ in $\sigma^{\prime}$. If $\lambda^{\prime}$ is a LIN gate which immediately feeds a MUL gate $\lambda_{0}^{\prime}$, then we set $\hat{\chi}\left(\lambda^{\prime}\right)=\hat{\chi}\left(\lambda_{0}^{\prime}\right)$. If $\lambda^{\prime}$ is an output node, then we let $\hat{\chi}\left(\lambda^{\prime}\right)=\hat{\mathbf{E}}[f]$. Finally, if $\lambda^{\prime}$ is a variable $x_{i}$, then we let $\hat{\chi}\left(\lambda^{\prime}\right)=\hat{C}_{i} /\left(1-\hat{\mathbf{E}}\left[x_{i}\right]\right)$. (Recall that $\hat{\mathbf{E}}\left[x_{i}\right]$ and $\hat{\mathbf{E}}[f]$ were both estimated in computing $\hat{C}_{i}$ and $\hat{T}_{i j}$.)

The assignments made above are actually performed by our reconstruction algorithm. For the sake of argument, we also define $\hat{\chi}(\lambda)$, for every node $\lambda$ of $\sigma$, to be $\hat{\chi}(\rho(\lambda))$.

We pause in our development to show that these estimates are fairly good. The following lemma will be helpful for this purpose. 
Lemma 12 Suppose MUL-gate $\Gamma_{i j}$ immediately feeds a trapped LIN gate which in turn immediately feeds muL-gate $\Gamma_{i k}$. Then $\left|E_{i j}-E_{i k}\right| \leq 18 \gamma$.

Proof: Since $\{i, j\}$ and $\{i, k\}$ are equivalent (by virtue of the trapped LIN gate), there must be some edge in $G_{1}$ directed from $\left\{i^{\prime}, k^{\prime}\right\}$ to $\left\{i^{\prime}, j^{\prime}\right\}$, and such that $\Gamma_{i^{\prime} j^{\prime}}$ feeds or is equal to $\Gamma_{i j}$, and $\Gamma_{i k}$ feeds or is equal to $\Gamma_{i^{\prime} k^{\prime}}$. By possibly renaming variables, we can assume without loss of generality that $\Gamma_{i j}=\Gamma_{i^{\prime} j}$ and $\Gamma_{i k}=\Gamma_{i^{\prime} k}$.

There cannot be a path in $G_{0}$ from $\left\{i^{\prime}, j^{\prime}\right\}$ to $\left\{i^{\prime}, k^{\prime}\right\}$ since this would deny the possibility of an edge in $G_{1}$ in the reverse direction.

In particular, this implies that there is no edge in $G_{0}$ from $\left\{i^{\prime}, j^{\prime}\right\}$ to $\left\{i^{\prime}, k^{\prime}\right\}$, meaning that the quotient test failed for this triple. As pointed out in Section 3.3.3, such a failure implies that

$$
\left|\hat{E}_{i^{\prime} j^{\prime}}-\hat{E}_{i^{\prime} k^{\prime}}\right| \leq 4 \gamma \text {. }
$$

Likewise, $G_{0}$ cannot include both of the edges $\left\langle\left\{i^{\prime}, j^{\prime}\right\},\left\{i^{\prime}, j\right\}\right\rangle$ and $\left\langle\left\{i^{\prime}, j\right\},\left\{i^{\prime}, k^{\prime}\right\}\right\rangle$, implying that either $\left|\hat{E}_{i^{\prime} j^{\prime}}-\hat{E}_{i^{\prime} j}\right|$ or $\left|\hat{E}_{i^{\prime} j}-\hat{E}_{i^{\prime} k^{\prime}}\right|$ is bounded by $4 \gamma$. Combined with the above argument, either case implies that

$$
\left|\hat{E}_{i^{\prime} j^{\prime}}-\hat{E}_{i^{\prime} j}\right| \leq 8 \gamma \text {. }
$$

A similar argument shows that

$$
\left|\hat{E}_{i^{\prime} j^{\prime}}-\hat{E}_{i^{\prime} k}\right| \leq 8 \gamma \text {. }
$$

Thus,

$$
\left|\hat{E}_{i^{\prime} j}-\hat{E}_{i^{\prime} k}\right| \leq 16 \gamma
$$

which implies by Lemma 6 that

$$
\left|E_{i j}-E_{i k}\right|=\left|E_{i^{\prime} j}-E_{i^{\prime} k}\right| \leq 18 \gamma .
$$

Lemma 13 Let $\lambda$ be a node of $\sigma$. Then $|\chi(\lambda)-\hat{\chi}(\lambda)| \leq 18 \gamma n$.

Proof: There are four cases to consider:

Case $1 \lambda$ is a variable $x_{i}$.

In this case, by Lemmas 5 and 11 ,

$$
\begin{aligned}
|\chi(\lambda)-\hat{\chi}(\lambda)| & =\left|\frac{C_{i}}{1-\mathbf{E}\left[x_{i}\right]}-\frac{\hat{C}_{i}}{1-\hat{\mathbf{E}}\left[x_{i}\right]}\right| \\
& \leq \frac{\left|C_{i}-\hat{C}_{i}\right|+|\chi(\lambda)| \cdot\left|\mathbf{E}\left[x_{i}\right]-\hat{\mathbf{E}}\left[x_{i}\right]\right|}{1-\hat{\mathbf{E}}\left[x_{i}\right]} \\
& \leq \frac{3 \tau+\tau}{\beta-\tau} \leq 18 \gamma n
\end{aligned}
$$


where we have made use of the assumed accuracy of $\hat{\mathbf{E}}\left[x_{i}\right]$ and $\hat{C}_{i}$, as well as the facts that $|\chi(\lambda)| \leq 1$, and that $1-\hat{\mathbf{E}}\left[x_{i}\right] \geq 1-\mathbf{E}\left[x_{i}\right]-\tau \geq\left|\hat{C}_{i}\right|-\tau \geq \beta-\tau$ (by equation (5)).

Case $2 \lambda$ is some MUL gate $\Gamma_{i j}$.

In this case, $\hat{\chi}(\lambda)=\hat{\chi}(\rho(\lambda))=\hat{E}_{k \ell}$ for some pair $k, \ell$ for which $\Gamma_{i j}^{\prime}=\Gamma_{k \ell}^{\prime}$ (i.e., $k, \ell$ was the pair chosen arbitrarily by our algorithm).

Without loss of generality, let $\Gamma_{i k}$ be the lowest common ancestor of $\Gamma_{i j}$ and $\Gamma_{k \ell}$. Then $\Gamma_{i k}^{\prime}=\Gamma_{i j}^{\prime}=\Gamma_{k \ell}^{\prime}$, and so every LIN gate is trapped on the paths from $\Gamma_{i j}$ to $\Gamma_{i k}$, and from $\Gamma_{k \ell}$ to $\Gamma_{i k}$. Applying Lemma 12 repeatedly to each of the $n_{1}$ MUL gates on the (inclusive) path from $\Gamma_{i j}$ to $\Gamma_{i k}$, we see that

$$
\left|E_{i k}-E_{i j}\right| \leq 18 \gamma\left(n_{1}-1\right) .
$$

Similarly,

$$
\left|E_{i k}-E_{k \ell}\right| \leq 18 \gamma\left(n_{2}-1\right)
$$

where $n_{2}$ is the number of MUL gates on the path from $\Gamma_{k \ell}$ to $\Gamma_{i k}$. Thus,

$$
\left|E_{i j}-\hat{E}_{k \ell}\right| \leq 18 \gamma\left(n_{1}+n_{2}-2\right)+\gamma .
$$

Since $n_{1}+n_{2}-1 \leq n$ (the maximum number of MUL gates in $f$ ), and since $\chi(\lambda)=E_{i j}$ and $\hat{\chi}(\lambda)=\hat{E}_{k \ell}$, the lemma follows in this case.

Case $3 \lambda$ is a LIN gate which feeds some MUL gate $\lambda_{0}$.

In this case, $\chi(\lambda)=\chi\left(\lambda_{0}\right)$, by Lemma 11 . Also, by definition,

$$
\hat{\chi}(\lambda)=\hat{\chi}(\rho(\lambda))=\hat{\chi}\left(\rho\left(\lambda_{0}\right)\right)=\hat{\chi}\left(\lambda_{0}\right)
$$

since $\rho(\lambda)$ immediately feeds $\rho\left(\lambda_{0}\right)$ in $\sigma^{\prime}$. Thus,

$$
|\chi(\lambda)-\hat{\chi}(\lambda)|=\left|\chi\left(\lambda_{0}\right)-\hat{\chi}\left(\lambda_{0}\right)\right|,
$$

and the lemma follows in this case by the proof for Case 2 .

Case $4 \lambda$ is the output LIN gate.

In this case, $\chi(\lambda)=\mathbf{E}[f]$, and $\hat{\chi}(\lambda)=\hat{\chi}(\rho(\lambda))=\hat{\mathbf{E}}[f]$, since $\rho(\lambda)$ is the output gate of $\sigma^{\prime}$. Thus,

$$
|\chi(\lambda)-\hat{\chi}(\lambda)|=|\mathbf{E}[f]-\hat{\mathbf{E}}[f]| \leq \tau \leq 18 \gamma n
$$

As mentioned, our reconstruction procedure works by visiting each node $\lambda$ of the given skeleton from the bottom up, starting with the input variables, estimating at 
each node the canonical form $h$ for the subformula $g$ subsumed by $\lambda$. An estimate $\hat{h}$ of $h$ is computed based on: (1) the node type of $\lambda,(2)$ the function $\hat{\chi}$, and (3) the previously computed estimates $\hat{h}_{i}$ of the canonical forms of the subformulas which immediately feed $\lambda$.

Specifically, the algorithm works as follows, depending on the node type of $\lambda$.

Case $1 \lambda$ is a variable $x_{i}$.

In this case, $g=x_{i}$, and $h=\mathbf{E}\left[\partial f / \partial x_{i}\right] \cdot x_{i}=\bar{B}_{i} x_{i}$. Motivated by equation (5), we estimate $\bar{B}_{i}$ using

$$
\hat{B}_{i}=\frac{\hat{C}_{i}}{\hat{\mathbf{E}}\left[x_{i}\right]\left(1-\hat{\mathbf{E}}\left[x_{i}\right]\right)}
$$

and we set $\hat{h}=\hat{B}_{i} x_{i}$.

Case $2 \lambda$ is a MUL gate.

In this case, $\lambda$ computes the product of $k$ subformulas whose canonical forms have previously been estimated by $\hat{h}_{1}, \ldots, \hat{h}_{k}$. (Although $f$ contains only binary MUL gates, we will want to apply this procedure to $\sigma^{\prime}$ which may include non-unary MUL gates.) In this case, we set

$$
\hat{h}=\frac{\hat{h}_{1} \cdots \hat{h}_{k}}{(\hat{\chi}(\lambda))^{k-1}} .
$$

(The motivation for this choice will be seen below.)

Case $3 \lambda$ is a LIN gate.

In this case, $\lambda$ computes a weighted sum of $k$ subformulas whose canonical forms were estimated by $\hat{h}_{1}, \ldots, \hat{h}_{k}$. Let $\lambda_{1}, \ldots, \lambda_{k}$ be the gates which immediately feed $\lambda$. Then we set

$$
\hat{h}=\hat{\chi}(\lambda)+\sum_{i=1}^{k}\left(\hat{h}_{i}-\hat{\chi}\left(\lambda_{i}\right)\right) .
$$

(Again, motivation will be provided below.)

The first fact we will want to prove about this procedure is that it yields the same result whether applied to $\sigma$ or $\sigma^{\prime}$. This is proved in the lemmas below.

We say that a node $\lambda$ in $\sigma$ is an apex-child of another node $\lambda_{0}$ if $\lambda$ is an apex node which feeds $\lambda_{0}$, and if there are no other apex nodes in $\sigma$ on the path from $\lambda$ to $\lambda_{0}$.

Also, we write $\hat{h}(\lambda)$ to denote the function $\hat{h}$ computed by the above procedure upon visiting gate $\lambda$. Here, it is understood that $\hat{h}(\lambda)$ was computed using the skeleton $\left(\sigma\right.$ or $\left.\sigma^{\prime}\right)$ to which $\lambda$ belongs. 
Lemma 14 Let $\lambda$ be a MUL gate or a trapped LIN gate of $\sigma$, and let $\lambda_{1}, \ldots, \lambda_{k}$ be its apex-children. Then

$$
\hat{h}(\lambda)=\frac{\hat{h}\left(\lambda_{1}\right) \cdots \hat{h}\left(\lambda_{k}\right)}{(\hat{\chi}(\lambda))^{k-1}} .
$$

Proof: By induction on the maximum depth of the apex-children (relative to $\lambda$ ). The lemma holds trivially in the degenerate base case that this depth is zero (i.e., $k=1$ ).

Suppose first that $\lambda$ is a trapped LIN gate immediately fed by MUL gate $\lambda_{0}$. By definition, $\rho(\lambda)=\rho\left(\lambda_{0}\right)$, so $\hat{\chi}(\lambda)=\hat{\chi}\left(\lambda_{0}\right)$. Thus, our procedure computes

$$
\hat{h}(\lambda)=\left(\hat{h}\left(\lambda_{0}\right)-\hat{\chi}\left(\lambda_{0}\right)\right)+\hat{\chi}(\lambda)=\hat{h}\left(\lambda_{0}\right) .
$$

The lemma then follows in this case by our inductive hypothesis on $\lambda_{0}$, and because $\lambda_{0}$ has the same apex-children as $\lambda$.

Suppose now that $\lambda$ is a MUL gate immediately fed by nodes $\Gamma_{1}$ and $\Gamma_{2}$. (Since $\lambda$ is in $\sigma$, it must be binary.) Then without loss of generality, $\lambda_{1}, \ldots, \lambda_{r}$ are the apexchildren of $\Gamma_{1}$, and $\lambda_{r+1}, \ldots, \lambda_{k}$ are the apex-children of $\Gamma_{2}$, for some $1 \leq r<k$. Then our procedure computes

$$
\hat{h}(\lambda)=\frac{\hat{h}\left(\Gamma_{1}\right) \cdot \hat{h}\left(\Gamma_{2}\right)}{\hat{\chi}(\lambda)}
$$

which, by inductive hypothesis, equals

$$
\frac{\hat{h}\left(\lambda_{1}\right) \cdots \hat{h}\left(\lambda_{k}\right)}{\hat{\chi}(\lambda) \cdot\left(\hat{\chi}\left(\Gamma_{1}\right)\right)^{r-1} \cdot\left(\hat{\chi}\left(\Gamma_{2}\right)\right)^{k-r-1}} .
$$

If $r>1$, then $\Gamma_{1}$ cannot be an apex gate, which implies that $\rho\left(\Gamma_{1}\right)=\rho(\lambda)$ and so $\hat{\chi}\left(\Gamma_{1}\right)=\hat{\chi}(\lambda)$. Thus, for $r \geq 1,\left(\hat{\chi}\left(\Gamma_{1}\right)\right)^{r-1}=(\hat{\chi}(\lambda))^{r-1}$. (Equality holds trivially when $r=1$.)

Similarly, for $r \leq k-1,\left(\hat{\chi}\left(\Gamma_{2}\right)\right)^{k-r-1}=(\hat{\chi}(\lambda))^{k-r-1}$. Thus, as desired,

$$
\hat{h}(\lambda)=\frac{\hat{h}\left(\lambda_{1}\right) \cdots \hat{h}\left(\lambda_{k}\right)}{(\hat{\chi}(\lambda))^{k-1}} .
$$

Lemma 15 Let $\lambda$ be an apex node of $\sigma$. Then $\hat{h}(\lambda)=\hat{h}(\rho(\lambda))$.

Proof: By induction on the depth of the subformula subsumed by $\lambda$. The result is trivial in the base case that $\lambda$ is a variable.

Suppose $\lambda$ is a LiN gate. Let $\lambda_{1}, \ldots, \lambda_{k}$ be the gates which immediately feed $\lambda$. Then each $\lambda_{i}$ is an apex node, so by inductive hypothesis, $\hat{h}\left(\lambda_{i}\right)=\hat{h}\left(\rho\left(\lambda_{i}\right)\right)$. Since the same computation is performed by the procedure in computing $\hat{h}(\lambda)$ and $\hat{h}(\rho(\lambda))$, the result follows trivially. 
Finally, suppose $\lambda$ is a MUL gate. Let $\lambda_{1}, \ldots, \lambda_{k}$ be the apex-children of $\lambda$. Then in $\sigma^{\prime}, \rho\left(\lambda_{1}\right), \ldots, \rho\left(\lambda_{k}\right)$ are exactly the gates that immediately feed $\rho(\lambda)$. Thus, by Lemma 14 and the inductive hypothesis, we have as desired

$$
\hat{h}(\lambda)=\frac{\hat{h}\left(\lambda_{1}\right) \cdots \hat{h}\left(\lambda_{k}\right)}{(\hat{\chi}(\lambda))^{k-1}}=\frac{\hat{h}\left(\rho\left(\lambda_{1}\right)\right) \cdots \hat{h}\left(\rho\left(\lambda_{k}\right)\right)}{(\hat{\chi}(\rho(\lambda)))^{k-1}}=\hat{h}(\rho(\lambda)) .
$$

Thus, in particular, since the output gate of $\sigma$ is an apex gate, Lemma 15 shows that the final formula computed by our procedure if applied to $\sigma$ would be the same as that computed for $\sigma^{\prime}$. Therefore, although the procedure would in fact be applied to $\sigma^{\prime}$, we will analyze its result as if it were computed using $\sigma$.

Suppose the procedure is visiting node $\lambda$, which subsumes subformula $g$ with canonical form $h$. The procedure computes an estimate $\hat{h}$ of $h$. For analysis purposes, we define an estimator $\hat{g}=\hat{h} / \mathbf{E}[\partial f / \partial g]$. We will prove, by induction, that

$$
\mathbf{E}[|g-\hat{g}|] \leq \frac{(2 s-1) \epsilon_{0}}{16 n}
$$

where $s$ is the number of nodes subsumed by $\lambda$ (inclusive). Since, when $\lambda$ is the output node, $f=g=h$ and $\hat{g}=\hat{h}$, and since an easy induction argument shows that there are at most $4 n-2$ nodes in the entire formula $f$, this will prove that the final output formula $\hat{f}$ is such that

$$
\mathbf{E}[|f-\hat{f}|] \leq \frac{(8 n-5) \epsilon_{0}}{16 n} \leq \frac{\epsilon}{2} .
$$

As usual, there are several cases to consider.

Case $1 \lambda$ is a variable $x_{i}$.

In this case, $g=x_{i}$, and, as noted above, $h=\bar{B}_{i} x_{i}$, and $\hat{h}=\hat{B}_{i} x_{i}$.

Then

$$
\mathbf{E}[|g-\hat{g}|]=\mathbf{E}\left[\left|x_{i}-\frac{\hat{B}_{i} x_{i}}{\bar{B}_{i}}\right|\right] \leq\left|1-\frac{\hat{B}_{i}}{\bar{B}_{i}}\right|=\frac{\left|\bar{B}_{i}-\hat{B}_{i}\right|}{\left|\bar{B}_{i}\right|} .
$$

Let $q=\mathbf{E}\left[x_{i}\right]\left(1-\mathbf{E}\left[x_{i}\right]\right)$ and let $\hat{q}=\hat{\mathbf{E}}\left[x_{i}\right]\left(1-\hat{\mathbf{E}}\left[x_{i}\right]\right)$. Then

$$
|q-\hat{q}| \leq 2\left|\mathbf{E}\left[x_{i}\right]-\hat{\mathbf{E}}\left[x_{i}\right]\right| \leq 2 \tau .
$$

Using Lemma 5, we have that (14) is at most

$$
\begin{aligned}
\frac{1}{\left|\bar{B}_{i}\right|} \cdot\left(\frac{\left|C_{i}-\hat{C}_{i}\right|}{\hat{q}}+\left|\bar{B}_{i}\right| \cdot \frac{|q-\hat{q}|}{\hat{q}}\right) & \leq \frac{3 \tau+2 \tau}{\left|\bar{B}_{i}\right| \cdot(q-2 \tau)} \\
& \leq \frac{5 \tau}{\left|C_{i}\right|-2 \tau} \leq \frac{10 \tau}{\beta} \leq \frac{\epsilon_{0}}{16 n}
\end{aligned}
$$


since $C_{i}=\bar{B}_{i} q$, and $2 \tau \leq \frac{1}{2} \beta \leq \frac{1}{2}\left|C_{i}\right|$. Thus, the inductive hypothesis is satisfied in this case since there is only one node subsumed by $\lambda$.

Case $2 \lambda$ is a MUL gate.

Since $\lambda$ is a MUL gate of $\sigma$, it must be binary, and so must be immediately fed by two nodes $\lambda_{1}$ and $\lambda_{2}$; these nodes subsume two subformulas $g_{1}$ and $g_{2}$ (respectively), so $g=g_{1} g_{2}$. Let $h_{1}$ and $h_{2}$ be the canonical forms of $g_{1}$ and $g_{2}$. We have that

$$
\begin{aligned}
h_{1} & =\mathbf{E}\left[\partial f / \partial g_{1}\right] \cdot g_{1} \\
& =\mathbf{E}[\partial f / \partial g] \cdot \mathbf{E}\left[\partial g / \partial g_{1}\right] \cdot g_{1} \\
& =\mathbf{E}[\partial f / \partial g] \cdot \bar{g}_{2} g_{1} .
\end{aligned}
$$

Similarly,

$$
h_{2}=\mathbf{E}[\partial f / \partial g] \cdot \bar{g}_{1} g_{2} .
$$

Thus,

$$
h=\mathbf{E}[\partial f / \partial g] \cdot g=\frac{h_{1} h_{2}}{\mathbf{E}[\partial f / \partial g] \cdot \bar{g}}=\frac{h_{1} h_{2}}{\chi(\lambda)} .
$$

Note that, if $\hat{h}_{1}$ and $\hat{h}_{2}$ are the previously computed estimates of $h_{1}$ and $h_{2}$, then our procedure computes

$$
\hat{h}=\frac{\hat{h}_{1} \hat{h}_{2}}{\hat{\chi}(\lambda)}
$$

For ease of notation, let $K=\mathbf{E}[\partial f / \partial g]$, let $e=\chi(\lambda)$ and let $\hat{e}=\hat{\chi}(\lambda)$. We would like to compute the error of $\hat{g}=\hat{h} / K$. We assume inductively that the estimates $\hat{g}_{1}$ and $\hat{g}_{2}$ are such that

$$
\mathbf{E}\left[\mid g_{i}-\hat{g}_{i} \|\right] \leq \frac{\left(2 s_{i}-1\right) \epsilon_{0}}{16 n}
$$

where $s_{i}$ is the number of nodes in $g_{i}$. By definition, $\hat{h}_{1}=K \bar{g}_{2} \hat{g}_{1}$ and $\hat{h}_{2}=K \bar{g}_{1} \hat{g}_{2}$. Thus,

$$
\begin{aligned}
\hat{g} & =\frac{\hat{h}_{1} \hat{h}_{2}}{K \hat{e}} \\
& =\frac{\left(K \bar{g}_{2} \hat{g}_{1}\right)\left(K \bar{g}_{1} \hat{g}_{2}\right)}{K \hat{e}} \\
& =\frac{K \bar{g}_{1} \bar{g}_{2}}{\hat{e}} \cdot \hat{g}_{1} \hat{g}_{2} \\
& =\frac{e}{\hat{e}} \cdot \hat{g}_{1} \hat{g}_{2} .
\end{aligned}
$$


So we can analyze the error of $\hat{g}$ as:

$$
\begin{aligned}
\mathbf{E}[|g-\hat{g}|] & =\mathbf{E}\left[\left|\frac{e}{\hat{e}} \hat{g}_{1} \hat{g}_{2}-g_{1} g_{2}\right|\right] \\
& \leq\left|1-\frac{e}{\hat{e}}\right| \cdot \mathbf{E}\left[\hat{g}_{1} \hat{g}_{2}\right]+\mathbf{E}\left[\left|g_{1} g_{2}-\hat{g}_{1} \hat{g}_{2}\right|\right] .
\end{aligned}
$$

We have that $|e-\hat{e}| \leq 18 \gamma n \leq \frac{1}{2} \beta^{2} \leq \frac{1}{2}|e|$ by Lemmas 11 and 13. Thus, the first term of (15) can be bounded by

$$
\begin{aligned}
\frac{|e-\hat{e}|}{|\hat{e}|} \cdot \mathbf{E}\left[\hat{g}_{1} \hat{g}_{2}\right] & \leq \frac{|e-\hat{e}|}{|e|-|e-\hat{e}|} \cdot \mathbf{E}\left[\hat{g}_{1} \hat{g}_{2}\right] \\
& \leq \frac{2|e-\hat{e}|}{|e|} \cdot \mathbf{E}\left[\hat{g}_{1} \hat{g}_{2}\right] \\
& \leq \frac{2|e-\hat{e}|}{|K| \bar{g}_{1} \bar{g}_{2}} \cdot\left(\bar{g}_{1} \bar{g}_{2}+\mathbf{E}\left[\left|g_{1} g_{1}-\hat{g}_{1} \hat{g}_{2}\right|\right]\right) .
\end{aligned}
$$

Also,

$$
\begin{aligned}
& \mathbf{E}\left[\left|g_{1} g_{2}-\hat{g}_{1} \hat{g}_{2}\right|\right] \leq \\
& \quad \bar{g}_{1} \cdot \mathbf{E}\left[\left|g_{2}-\hat{g}_{2}\right|\right]+\bar{g}_{2} \cdot \mathbf{E}\left[\left|g_{1}-\hat{g}_{1}\right|\right]+\mathbf{E}\left[\left|g_{1}-\hat{g}_{1}\right|\right] \cdot \mathbf{E}\left[\left|g_{2}-\hat{g}_{2}\right|\right] .
\end{aligned}
$$

Thus, expression (16) is at most

$$
2|e-\hat{e}| \cdot\left(\frac{1}{|K|}+\frac{\mathbf{E}\left[\left|g_{1}-\hat{g}_{1}\right|\right]}{|K| \bar{g}_{1}}+\frac{\mathbf{E}\left[\left|g_{2}-\hat{g}_{2}\right|\right]}{|K| \bar{g}_{2}}+\frac{\mathbf{E}\left[\left|g_{1}-\hat{g}_{1}\right|\right] \cdot \mathbf{E}\left[\left|g_{2}-\hat{g}_{2}\right|\right]}{\cdot|K| \bar{g}_{1} \bar{g}_{2}}\right) .
$$

Note that

$$
|K|=|\mathbf{E}[\partial f / \partial g]| \geq\left|\mathbf{E}\left[\partial f / \partial x_{i}\right]\right| \geq\left|C_{i}\right| \geq \beta,
$$

where $x_{i}$ is any variable relevant to $g$. Likewise, $\left|K \bar{g}_{1}\right|=\left|\mathbf{E}\left[\partial f / \partial g_{2}\right]\right| \geq \beta$, and similarly, $\left|K \bar{g}_{2}\right| \geq \beta$.

Combining facts and rearranging terms, this shows that expression (15) is at most

$$
\begin{aligned}
\frac{36 \gamma n}{\beta}+ & \left(1+\frac{36 \gamma n}{\beta}\right) \cdot\left(\mathbf{E}\left[\left|g_{1}-\hat{g}_{1}\right|\right]+\mathbf{E}\left[\left|g_{2}-\hat{g}_{2}\right|\right]\right) \\
& +\left(1+\frac{36 \gamma n}{\beta^{2}}\right) \cdot \mathbf{E}\left[\left|g_{1}-\hat{g}_{1}\right|\right] \mathbf{E}\left[\left|g_{2}-\hat{g}_{2}\right|\right] \\
\leq & \frac{36 \gamma n}{\beta}+\left(1+\frac{36 \gamma n}{\beta}\right) \cdot \frac{\left(2 s_{1}+2 s_{2}-2\right) \epsilon_{0}}{16 n} \\
& +\left(1+\frac{36 \gamma n}{\beta^{2}}\right) \cdot\left(2 s_{1}\right)\left(2 s_{2}\right) \cdot\left(\frac{\epsilon_{0}}{16 n}\right)^{2} \\
\leq & \frac{(2 s-1) \epsilon_{0}}{16 n}+\frac{36 \gamma n}{\beta} \cdot\left(1+\frac{s \epsilon_{0}}{8 n}\right)+\frac{s_{1} s_{2} \epsilon_{0}^{2}}{32 n^{2}}-\frac{3 \epsilon_{0}}{16 n}
\end{aligned}
$$

where $s=s_{1}+s_{2}+1$ is the number of nodes in $g$, and where we have used the fact that $36 \gamma n \leq \beta^{2}$. We have that 


$$
\frac{36 \gamma n}{\beta} \cdot\left(1+\frac{s \epsilon_{0}}{8 n}\right) \leq \frac{3 \cdot 36 \gamma n}{2 \beta}=\frac{3 \cdot 18 \cdot 28 \cdot 5^{3} \epsilon_{0}}{20^{5} n} \leq \frac{\epsilon_{0}}{16 n} .
$$

Also, since $s_{1}+s_{2} \leq s \leq 4 n$, and since $\epsilon_{0} \leq 1 / n$,

$$
\frac{s_{1} s_{2} \epsilon_{0}^{2}}{32 n^{2}} \leq \frac{4 n^{2} \epsilon_{0}^{2}}{32 n^{2}}=\frac{\epsilon_{0}^{2}}{8} \leq \frac{\epsilon_{0}}{8 n} .
$$

Combined with (17), these facts show that

$$
\mathbf{E}[|g-\hat{g}|] \leq \frac{(2 s-1) \epsilon_{0}}{16 n}
$$

as desired.

Case $3 \lambda$ is a LIN gate.

In this case, the gate $\lambda$ is fed by $k$ nodes $\lambda_{1}, \ldots, \lambda_{k}$. These nodes subsume subformulas $g_{1}, \ldots, g_{k}$, each $g_{i}$ having canonical form $h_{i}$, estimated by $\hat{h}_{i}$. Let $s_{i}$ be the number of nodes in $g_{i}$. Then by inductive hypothesis,

$$
\mathbf{E}\left[\left|g_{i}-\hat{g}_{i}\right|\right] \leq \frac{\left(2 s_{i}-1\right) \epsilon_{0}}{16 n}
$$

where, as usual, $\hat{g}_{i}=\hat{h}_{i} / \mathbf{E}\left[\partial f / \partial g_{i}\right]$.

Let $K=\mathrm{E}[\partial f / \partial g]$, and suppose that $g=z+\sum w_{i} g_{i}$ where $z$ and the $w_{i}$ 's are constants. (If not otherwise specified, it is understood here and below that all summations are for $i=1, \ldots, k$.) Then

$$
\begin{aligned}
h_{i} & =\mathbf{E}\left[\partial f / \partial g_{i}\right] \cdot g_{i} \\
& =\mathbf{E}[\partial f / \partial g] \cdot \mathbf{E}\left[\partial g / \partial g_{i}\right] \cdot g_{i}=K w_{i} g_{i} .
\end{aligned}
$$

Thus,

$$
\begin{aligned}
h & =\mathbf{E}[\partial f / \partial g] \cdot g=K g \\
& =K z+\sum K w_{i} g_{i}=K z+\sum h_{i} .
\end{aligned}
$$

Since $\chi\left(\lambda_{i}\right)=\mathbf{E}\left[h_{i}\right]$ and $\chi(\lambda)=\mathbf{E}[h]$, this equation implies that

$$
K z=\mathbf{E}\left[h-\sum h_{i}\right]=\chi(\lambda)-\sum \chi\left(\lambda_{i}\right)
$$

and so

$$
h=\chi(\lambda)+\sum\left(h_{i}-\chi\left(\lambda_{i}\right)\right) .
$$

This is the motivation for our algorithm estimating $h$ by

$$
\hat{h}=\hat{\chi}(\lambda)+\sum\left(\hat{h}_{i}-\hat{\chi}\left(\lambda_{i}\right)\right) .
$$


Recall that $\hat{h}=K \hat{g}$ and $\hat{h}_{i}=K w_{i} \hat{g}_{i}$. Thus,

$$
\hat{g}=\frac{\hat{\chi}(\lambda)-\sum \hat{\chi}\left(\lambda_{i}\right)}{K}+\sum w_{i} \hat{g}_{i}
$$

and so, letting $s=1+\sum s_{i}$ be the number of nodes in $g$, we have

$$
\begin{aligned}
\mathbf{E}[|g-\hat{g}|] \leq & \frac{1}{|K|} \cdot\left(\mathbf{E}[\mid \chi(\lambda)-\hat{\chi}(\lambda) \|]+\sum \mathbf{E}\left[\left|\chi\left(\lambda_{i}\right)-\hat{\chi}\left(\lambda_{i}\right)\right|\right]\right) \\
& +\sum\left|w_{i}\right| \cdot \mathbf{E}\left[\left|g_{i}-\hat{g}_{i}\right|\right] \\
\leq & \frac{18 \gamma n(1+k)}{|K|}+\sum \frac{\left(2 s_{i}-1\right) \epsilon_{0}}{16 n} \\
\leq & \frac{(2 s-1) \epsilon_{0}}{16 n}+\frac{18 \gamma n(1+k)}{\beta}-\frac{(1+k) \epsilon_{0}}{16 n} \\
\leq & \frac{(2 s-1) \epsilon_{0}}{16 n}
\end{aligned}
$$

as desired.

Combined with the previous cases, this completes the induction, and proves that the final computed hypothesis $\hat{f}$ has error at most $\mathbf{E}[|f-\hat{f}|] \leq \epsilon_{0} / 2 \leq \epsilon / 2$.

\subsection{Putting it all together}

Thus, we showed in Stage I how to eliminate uncorrelated variables from the target formula $f$, yielding another formula $f_{\mathrm{I}}$ with $\mathbf{E}\left[\left|f-f_{\mathrm{I}}\right|\right] \leq \epsilon / 2$.

In Stage II, we regarded $f_{\mathrm{I}}$ as the target, and showed how to identify gate types, and how to find a skeleton $\sigma^{\prime}$ of $f_{\mathrm{I}}$ that closely approximates the true skeleton $\sigma$.

Finally, in Stage III, we described a procedure for filling in the missing LIN-gate parameters. In particular, we showed that this procedure yields the same result whether applied to the true skeleton $\sigma$, or its approximation $\sigma^{\prime}$. Then we showed that when applied to $\sigma$, the result $\hat{f}$ is such that $\mathbf{E}\left[\left|f_{\mathrm{I}}-\hat{f}\right|\right] \leq \epsilon / 2$. It follows immediately that $\mathbf{E}[|f-\hat{f}|] \leq \epsilon$ as desired. In other words, $\hat{f}$ is an $\epsilon$-good model of probability for $f$ with probability at least $1-\delta$.

All of the operations described are clearly polynomial time, and the sample size is also polynomial. The sample was needed to estimate various expectations. For the accuracy needed, we can use Hoeffding's (1963) Inequality to show that a sample of size $O\left(\log (n / \delta) / \tau^{2}\right)$ suffices.

After the sample is drawn our algorithm records the obtained estimates of these values. All of the remaining operations of the algorithm take negligible time compared to the time needed to compute and record these values from the sample.

Thus we have: 
Theorem 1 There exists an algorithm with the following properties: Given $\epsilon, \delta>0$ and access to random examples chosen according to a product distribution and classified randomly according to some read-once real formula $f$, the algorithm outputs an $\epsilon$-good model of probability for $f$ with probability at least $1-\delta$. The sample size needed by the algorithm is

$$
O\left(\frac{n^{10}}{\epsilon^{6}} \cdot\left(n+\frac{1}{\epsilon}\right)^{2} \cdot \log (n / \delta)\right)
$$

and its running time is

$$
O\left(\frac{n^{12}}{\epsilon^{6}} \cdot\left(n+\frac{1}{\epsilon}\right)^{2} \cdot \log (n / \delta)\right)
$$

From the comments made in Section 2, we have as corollary:

Corollary 1 There exists a polynomial-time algorithm that PAC-learns the class of read-once Boolean formulas against any product distribution.

Finally, we remark briefly that the algorithm given in this paper, though analyzed only for Boolean variables, can in fact be modified to handle real-valued variables with range in $[0,1]$. Note that if $x_{i}$ is not Boolean, then equation (5) may not hold. However, the given derivation of that equation does show that, in general,

$$
C_{i}=\bar{B}_{i} \cdot \operatorname{var}\left(x_{i}\right)
$$

where $\operatorname{var}\left(x_{i}\right)$ is simply the variance of $x_{i}$ :

$$
\operatorname{var}\left(x_{i}\right)=\mathbf{E}\left[\left(x_{i}-\bar{x}_{i}\right)^{2}\right]=\mathbf{E}\left[x_{i}^{2}\right]-\left(\mathbf{E}\left[x_{i}\right]\right)^{2} .
$$

Similarly, equation (9) becomes

$$
T_{i j}=\bar{A}_{i j} \cdot \operatorname{var}\left(x_{i}\right) \cdot \operatorname{var}\left(x_{j}\right) .
$$

Using these modified versions of equations (5) and (9) we can adjust the rest of the proof appropriately to derive a similar learnability result for non-Boolean variables (with some degradation in the time and sample complexity).

\section{Conclusion and open problems}

In this paper, we have described a polynomial-time algorithm for learning a class of probabilistic read-once formulas. The algorithm reconstructs an approximation of the formula using empirical estimates of various statistical measures.

The main open question is to determine how far this apparently powerful method can be extended. For example, can such a technique be used to learn read-once 
formulas over other kinds of gates, such as parity or threshold gates? Can our method be applied to formulas which are not read-once? Can it be extended beyond product distributions? Can it be extended to the so-called two-oracie model? Are there other classes entirely different to which it might be extended, such as decision trees, or finite automata?

Considering the class discussed in this paper, can the algorithm used (or its analysis) be significantly improved? Turning the question around, can we find good, nontrivial lower bounds for such distribution-specific learning problems? It is unclear what such a lower-bound proof would look like, especially since, in the PAC model, much smaller sample sizes are known to suffice in a computationally unbounded setting. (This follows, for instance, from Occam's Razor of Blumer et al. (1987).)

\section{Acknowledgements}

Thanks to Ron Rivest for his comments and careful reading of an earlier draft of this material, and to Sally Goldman, Tom Hancock, Lisa Hellerstein and Yishay Man-

sour for several helpful discussions. I am also grateful to two anonymous referees for their thorough reading and thoughtful suggestions.

This paper was prepared in part while I was at Harvard University with the support of AFOSR Grant 89-0506, and also while I was at the MIT Laboratory for Computer Science with the support of ARO Grant DAAL03-86-K-0171, DARPA Contract N00014-89-J-1988, and a grant from the Siemens Corporation.

\section{References}

Angluin, D., Hellerstein, L., and Karpinski, M. (1993). Learning read-once formulas with queries. Journal of the Association for Computing Machinery, 40(1):185-210.

Angluin, D. and Laird, P. (1988). Learning from noisy examples. Machine Learning, 2(4):343-370. Blumer, A., Ehrenfeucht, A., Haussler, D., and Warmuth, M. K. (1987). Occam's razor. Information Processing Letters, 24(6):377-380.

Bshouty, N. H., Hancock, T. R., and Hellerstein, L. (1992). Learning arithmetic read-once formulas. In Proceedings of the Twenty-Fourth Annual ACM Symposium on the Theory of Computing, pages $370-381$.

Furst, M. L., Jackson, J. C., and Smith, S. W. (1991). Improved learning of $A C^{0}$ functions. In Proceedings of the Fourth Annual Workshop on Computational Learning Theory, pages 317-325.

Goldman, S. A., Kearns, M. J., and Schapire, R. E. (1990). Exact identification of circuits using fixed points of amplification functions. In 31st Annual Symposium on Foundations of Computer Science, pages 193-202. To appear, SIAM Journal on Computing.

Hancock, T. and Hellerstein, L. (1991). Learning read-once formulas over fields and extended bases. In Proceedings of the Fourth Annual Workshop on Computational Learning Theory, pages $326-336$.

Hancock, T. and Mansour, Y. (1991). Learning monotone $k \mu$ DNF formulas on product distributions. In Proceedings of the Fourth Annual Workshop on Computational Learning Theory, pages 179-183.

Hancock, T. R. (1990). Identifying $\mu$-formula decision trees with queries. In Proceedings of the Third Annual Workshop on Computational Learning Theory, pages 23-37. 
Hellerstein, L. and Karpinski, M. (1990). Read-once formulas over different bases. Technical Report 8556-CS, University of Bonn.

Hoeffding, W. (1963). Probability inequalities for sums of bounded random variables. Journal of the American Statistical Association, 58(301):13-30.

Kearns, M., Li, M., Pitt, L., and Valiant, L. (1987). On the learnability of Boolean formulae. In Proceedings of the Nineteenth Annual ACM Symposium on Theory of Computing, pages 285-295.

Kearns, M. and Valiant, L. G. (1989). Cryptographic limitations on learning Boolean formulae and finite automata. In Proceedings of the Twenty First Annual ACM Symposium on Theory of Computing, pages 433-444. To appear, Journal of the Association for Computing Machinery.

Kearns, M. J. and Schapire, R. E. (1990). Efficient distribution-free learning of probabilistic concepts. In 31st Annual Symposium on Foundations of Computer Science, pages 382-391. To appear, Journal of Computer and System Sciences.

Linial, N., Mansour, Y., and Nisan, N. (1989). Constant depth circuits, Fourier transform, and learnability. In 30th Annual Symposium on Foundations of Computer Science, pages 574-579.

Pagallo, G. and Haussler, D. (1989). A greedy method for learning $\mu \mathrm{DNF}$ functions under the uniform distribution. Technical Report UCSC-CRL-89-12, University of California Santa Cruz, Computer Research Laboratory.

Sloan, R. H. (1988). Types of noise in data for concept learning. In Proceedings of the 1988 Workshop on Computational Learning Theory, pages 91-96.

Valiant, L. G. (1984). A theory of the learnable. Communications of the ACM, 27(11):1134-1142.

Verbeurgt, K. (1990). Learning DNF under the uniform distribution in quasi-polynomial time. In Proceedings of the Third Annual Workshop on Computational Learning Theory, pages 314-326.

Yamanishi, K. (1992). A learning criterion for stochastic rules. Machine Learning, 9(2/3):165203. 